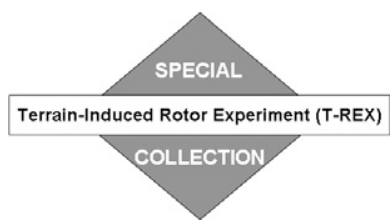

\title{
Observations and Numerical Simulations of Subrotor Vortices during T-REX
}

\author{
James D. Doyle, ${ }^{*}$ VAnda Grubišić, ${ }^{+}$William O. J. Brown, ${ }^{\#}$ Stephan F. J. De Wekker, ${ }^{@}$

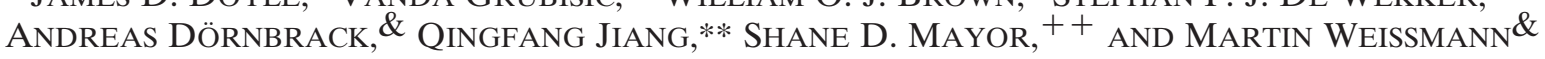 \\ * Naval Research Laboratory, Monterey, California \\ + Desert Research Institute, Reno, Nevada \\ \# NCAR, Boulder, Colorado \\ @ University of Virginia, Charlottesville, Virginia \\ \& Deutsches Zentrum für Luft- und Raumfahrt, Institut für Physik der Atmosphäre, Oberpfaffenhofen, Germany \\ **UCAR, Monterey, California \\ ++ California State University, Chico, California
}

(Manuscript received 26 August 2008, in final form 2 November 2008)

\begin{abstract}
High-resolution observations from scanning Doppler and aerosol lidars, wind profiler radars, as well as surface and aircraft measurements during the Terrain-induced Rotor Experiment (T-REX) provide the first comprehensive documentation of small-scale intense vortices associated with atmospheric rotors that form in the lee of mountainous terrain. Although rotors are already recognized as potential hazards for aircraft, it is proposed that these small-scale vortices, or subrotors, are the most dangerous features because of strong wind shear and the transient nature of the vortices. A life cycle of a subrotor event is captured by scanning Doppler and aerosol lidars over a 5-min period. The lidars depict an amplifying vortex, with a characteristic length scale of $\sim 500-1000 \mathrm{~m}$, that overturns and intensifies to a maximum spanwise vorticity greater than $0.2 \mathrm{~s}^{-1}$. Radar wind profiler observations document a series of vortices, characterized by updraft/downdraft couplets and regions of enhanced reversed flow, that are generated in a layer of strong vertical wind shear and subcritical Richardson number. The observations and numerical simulations reveal that turbulent subrotors occur most frequently along the leading edge of an elevated sheet of horizontal vorticity that is a manifestation of boundary layer shear and separation along the lee slopes. As the subrotors break from the vortex sheet, intensification occurs through vortex stretching and in some cases tilting processes related to threedimensional turbulent mixing. The subrotors and ambient vortex sheet are shown to intensify through a modest increase in the upstream inversion strength, which illustrates the predictability challenges for the turbulent characterization of rotors.
\end{abstract}

\section{Introduction}

Aircraft pilots have long known of the potential for hazardous weather near and above mountains. Of these potential hazards, atmospheric rotors are considered among the most severe due to very strong lower tropospheric turbulence and shear and have contributed to numerous incidents and accidents worldwide (e.g., Carney et al. 1996; Darby and Poulos 2006). Rotors are characterized by strong gusty downslope winds near the surface that decelerate rapidly in the lee of mountains, transitioning to weaker and unsteady flow often di-

Corresponding author address: James D. Doyle, Naval Research Laboratory, Marine Meteorology Division, 7 Grace Hopper Ave., Monterey, CA 93943-5502.

E-mail: james.doyle@nrlmry.navy.mil rected back toward the mountain (e.g., Holmboe and Klieforth 1957; Kuettner 1959; Doyle and Durran 2002). Although there has been considerable anecdotal evidence of the ferocious nature of the turbulence associated with rotors (Holmboe and Klieforth 1957; Kahn et al. 1997; National Transportation Safety Board 1992; Whelan 2000; Bond et al. 2006), the internal structure of rotors and associated turbulence has largely been unexplored. Recent results from very high-resolution numerical simulations presented in Doyle and Durran (2007) reveal the presence of turbulent, small-scale vortices that they refer to as subrotors, which may pose a significant aviation hazard because of their intensity. However, the existence of subrotor vortices has never been observationally confirmed as sufficient quantitative measurements to document even the basic characteristics of subrotors have been lacking. 
The Sierra Wave Project and its follow-on, the Jet Stream Project, both of which took place in the early 1950s (Holmboe and Klieforth 1957; Grubišić and Lewis 2004), were the first coordinated research efforts focused on observing topographically forced phenomena and documented several research aircraft penetrations of rotors and associated turbulence (Holmboe and Klieforth 1957). The relatively rare in situ research aircraft encounters with rotors (Lester and Fingerhut 1974), along with occasional lidar observations of downslope windstorms (Banta et al. 1990; Weissmann et al. 2004), all point to the potential for extreme turbulence that is likely composed of intense smaller-scale vortices, consistent with the notion of subrotor vortices. Unfortunately, the spatial and temporal scales of the turbulence and shear associated with rotors and subrotors are sufficiently small to elude conventional meteorological observing systems and weather predictions, further exacerbating the aviation risk.

The recent Terrain-induced Rotor Experiment (T-REX), which is a coordinated international effort focused on exploring the structure and evolution of atmospheric rotors and associated phenomena in complex terrain (Grubišić et al. 2008), has brought together for the first time a comprehensive suite of remote sensing and aircraft observations to document the characteristics of subrotors, rotors, and mountain waves generated by flow over terrain. One of the primary objectives of T-REX is to study the internal dynamics of rotors and their interaction with boundary layer processes near the earth's surface. The T-REX field campaign was centered on Owens Valley in the lee of the southern Sierra Nevada in eastern California. This portion of the Sierra Nevada is the tallest quasi-two-dimensional topographic barrier in the contiguous United States and includes the highest peak in the lower 48 states and the steepest lee slopes ( $\sim 30 \%$ grade). The ground-based instrumentation in Owens Valley included a scanning aerosol lidar, two Doppler lidars, and three radar wind profilers. T-REX marks the first time such a suite of ground-based systems has been deployed to observe mountain waves and rotors.

Rotors have been observed at numerous locations around the world such as the Rocky Mountains (Lester and Fingerhut 1974; Darby and Poulos 2006), the Wickham Range on the Falkland Islands (Mobbs et al. 2005), and the Sierra Nevada range (Holmboe and Klieforth 1957; Kuettner 1959; Grubišić and Billings 2007). The Sierra Rotors Project (SRP), which took place prior to T-REX in the spring of 2004, consisted of a suite of ground-based observing platforms upstream and downstream of the Sierra Nevada range. Several events during SRP were documented with accelerated downslope flow along the lee slope together with reversed flow and rotors in the lee of the Sierra (Grubišić and Billings 2007). Downslope windstorms during SRP were found to occur most frequently in the local afternoons owing to diurnal boundary layer heating effects that are manifested as a multiscale dynamical response (Jiang and Doyle 2008).

Numerical simulations (Doyle and Durran 2002; Vosper 2004; Hertenstein and Kuettner 2005) and observational studies (Mobbs et al. 2005) have suggested that a crucial aspect of rotor development involves the mutual interaction between the mountain lee wave and surface boundary layer. A thin vortex sheet, generated by mechanical shear in the boundary layer, separates from the surface due to adverse pressure gradients associated with lee waves. The two-dimensional simulations of Doyle and Durran (2002) indicate that the magnitude of the reversed flow within the rotor for a simulation with surface friction is well correlated with the strength of the adverse pressure gradient in an otherwise identical simulation without surface friction, which highlights the synergy between the boundary layer and lee wave. The link between rotors and mountain wave amplitude is also supported by the numerical simulations of Vosper (2004), which demonstrate that, as the lee wave amplifies, a critical threshold for the onset of flow separation is exceeded and rotor development ensues. The two-dimensional simulations of Hertenstein and Kuettner (2005) were used to explore two distinct flow states that they refer to as type- 1 and type- 2 rotors, distinguished by the vertical shear within low-level inversions.

Very high resolution 3D numerical simulations of rotors (Doyle and Durran 2007) reveal the formation of intense vortices or subrotors along an elevated sheet of horizontal vorticity, particularly along the lee wave updraft where the vertical and horizontal wind shear and turbulence production are maximized. Parallel shear instability breaks this vortex sheet into small intense subrotor vortices. These subrotor circulations may pose the greatest hazard to aviation and are most challenging to observe because of their transient nature. Ultimately, it is these simulations that have served as one of the primary motivations for T-REX: to search for subrotors and document their characteristics.

In this study, we examine Intensive Observation Period (IOP) 13 of T-REX, which featured a strong synopticscale system that impinged on the Sierra Nevada range and resulted in large-amplitude mountain waves, rotors, and coherent turbulent eddies in the lee of the Sierras. The primary objectives of this study are to (i) advance our understanding of the internal structure of rotors through an analysis of the T-REX observations, (ii) explore the 


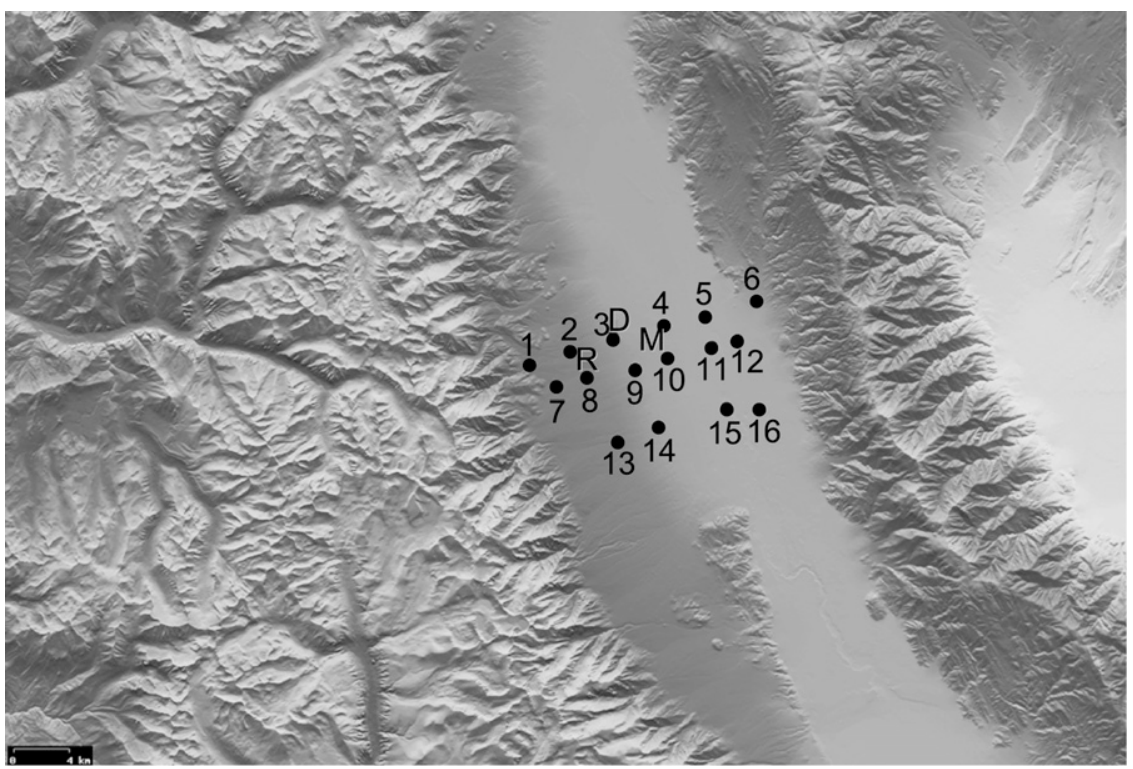

FIG. 1. Locations of the individual stations in the DRI surface network are shown by the solid circles: The location of the REAL lidar (R), the DLR Doppler lidar (D), and the MAPR radar wind profiler (M) are shown. The terrain relief is shaded corresponding to the central Sierra Nevada and Owens Valley regions. The DRI surface stations identification numbers are shown as well.

T-REX observations for evidence of subrotor-scale vortices, and (iii) provide further insight into the subrotor characteristics through the use of high-resolution numerical simulations. In the following section an overview of the T-REX instrumentation used in this study is presented. The numerical model is described in section 3. Section 4 presents an analysis of the observations during IOP 13 with an emphasis on the subrotor vortices. The results from high-resolution model simulations are described in section 5. The summary and conclusions are presented in section 6 .

\section{T-REX instrumentation}

In this study, we make use of the extensive T-REX ground-based network, which was primarily located near Independence, CA (Fig. 1). The ground-based system included two Doppler lidars, one aerosol lidar, three sodars and Radio Acoustic Sounding Systems (RASS), and three radar (915 MHz) wind profilers [of which one was mobile; all are part of the National Center for Atmospheric Research (NCAR) Integrated Sounding Systems (ISS)]. Airborne dust with a source from the dry Owens Lake bed improves the lidar capacity to measure the attendant flow and associated small-scale circulations, eddies, and turbulence, as was the case during IOP 13 . We focus on three ground-based remote sensing systems-a Doppler lidar, an aerosol lidar, and a radar wind profiler-along with radiosonde observations and the Desert Research Institute (DRI) surface observational network to document the rotor structure and characteristics during IOP 13. The Deutsches Zentrum für Luft- und Raumfahrt (DLR) Institut für Physik der Atmosphäre operated a coherent 2- $\mu \mathrm{m}$ highresolution Doppler lidar during T-REX to examine the structure and turbulence characteristics of rotors. The lidar has an along-beam resolution of $105 \mathrm{~m}$ and a refresh rate for a full vertical scan that was in the range of 30-90 s. The NCAR Raman-Shifted Eye-Safe Aerosol lidar (REAL) system (Mayor et al. 2007) has a rapidtemporal and high-spatial resolution scanning capability (backscatter intensity measured at 3-m range intervals with a 30 -s refresh rate for vertical scans) that made it particularly valuable during T-REX (De Wekker and Mayor 2009). The REAL lidar was located approximately $3 \mathrm{~km}$ west-southwest of the Doppler lidar along the alluvial slope of the eastern Sierras (Fig. 1). The NCAR Multiple Antenna Profiler Radar (MAPR) (Cohn et al. 2001) is an advanced 915-MHz wind profiler radar that uses four vertically pointing subarrays to make rapid wind measurements of the lower atmosphere on time scales of $30 \mathrm{~s}$ to $5 \mathrm{~min}$-considerably faster than the 10-15 min required by traditional wind profilers. The MAPR was located in Owens Valley $2 \mathrm{~km}$ east of the DLR Doppler lidar (Fig. 1). A network of 16 automatic weather stations (AWS) was installed by 
DRI in the valley and is configured in three nearly parallel lines spanning across the valley (Fig. 1). Each station consists of a standard $30-\mathrm{ft}(10-\mathrm{m})$ meteorological tower and sensors for wind, temperature, relative humidity, and pressure. The sensors are sampled every $3 \mathrm{~s}$, and the data is temporally averaged over 30-s nonoverlapping intervals. The weather stations are referred to by sequential numbers in the west-east segments, beginning with the northwestern-most station. Upper-air soundings were made from two GPS radiosonde launch sites in the valley near Independence. On the west side of the Sierra Nevada, a fixed and a mobile GPS radiosondes site, a thermosonde, and a K-band radar were deployed to monitor the incoming flow characteristics. A more detailed description of the instrumentation can be found in Grubišić et al. (2008).

Three research aircraft were used in the T-REX campaign to document the mountain wave-rotor coupled system over Owens Valley and to provide kinematic and thermodynamic information about airflow upstream and downstream of the Sierra Nevada range. The T-REX aircraft included the National Science Foundation (NSF)-NCAR High-Performance Instrumented Airborne Platform for Environmental Research (HIAPER), the U.K. FAAM BAe146 (which was deployed during March 17-April 9 and was not available during IOP 13), and the University of Wyoming King Air (UWKA). The three aircraft covered a range of altitudes from near the surface within Owens Valley to approximately $14 \mathrm{~km}$ MSL. The focus of this study is on the low-level rotor structure and associated turbulence in the valley; therefore, only the King Air flight-level dataset is used in this study to augment the analysis of the ground-based instrumentation.

\section{Numerical model description}

The atmospheric portion of the Naval Research Laboratory's Coupled Ocean-Atmospheric Mesoscale Prediction System (COAMPS) (Hodur 1997) is used to conduct idealized three-dimensional simulations based on the conditions during T-REX. The model is formulated using a finite-difference approximation to the fully compressible, nonhydrostatic equations and uses a terrain-following vertical coordinate transformation. The horizontal advection and horizontal smoothing terms are represented by fourth-order accurate differencing, and second-order differencing is used to represent the vertical advection, pressure gradient, and divergence terms. A Robert time filter is applied to all predicted variables. A time splitting technique with a semiimplicit formulation for the vertical acoustic modes is used to efficiently integrate the compressible equations (Klemp and Wilhelmson 1978). The model formulation and equation set are discussed in detail in Doyle and Durran (2007). Terrain is incorporated through a transformation to the following coordinate:

$$
\sigma=\frac{z_{t}(z-h)}{z_{t}-h}
$$

where $z_{t}$ is the depth of the model computation domain, $z$ is the physical height, $h$ is the terrain elevation, and $\sigma$ is the transformed vertical coordinate.

The subgrid-scale turbulent flux parameterization is based on Lilly (1962) and is described in Golaz et al. (2005) and Doyle and Durran (2007). The surface ground temperature is assumed to be in balance with the surface air temperature, resulting in a zero heat flux for these experiments. The surface momentum flux is computed following the Louis (1979) and Louis et al. (1982) formulation, which makes use of Monin-Obukhov similarity theory and assumes a vegetation roughness length of $1 \mathrm{~cm}$.

A radiation condition is used for the lateral boundaries following Orlanski (1976), with the exception that the Doppler-shifted phase speed $(u \pm c)$ is specified and temporally invariant at each boundary (Pearson 1974; Durran et al. 1993). The mitigation of reflected waves from the upper boundary is accomplished through a radiation condition formulated following the Durran (1999) approximation to the Klemp and Durran (1983) and Bougeault (1983) techniques.

The simulations are initialized using a reference state based on the conditions upstream of the Sierra Nevada during T-REX IOP 13 through the use of a radiosonde launched west of the Sierra at 2100 UTC 16 April, shown in Fig. 2. The cross-mountain wind component increases throughout the troposphere from roughly $3 \mathrm{~m} \mathrm{~s}^{-1}$ near the surface to $53 \mathrm{~m} \mathrm{~s}^{-1}$ at the tropopause and decreases to an approximate mean-state critical level near the domain top. The near-surface potential temperature profile exhibits a neutral layer that extends up to $1.5 \mathrm{~km}$. A layer of enhanced static stability is present at $3-4.5 \mathrm{~km}$ MSL characterized by a Brunt-Väisälä frequency $N$ $0.016 \mathrm{~s}^{-1}$, where $N=[(g / \theta)(\partial \theta / \partial z)]^{1 / 2}$. The wind profile is smoothed below $5 \mathrm{~km}$ to eliminate small-scale shear layers and the minimum mountain-normal wind component in the boundary layer is $5 \mathrm{~m} \mathrm{~s}^{-1}$. The model is initialized with small random and spatially uncorrelated potential temperature perturbations of maximum magnitude, $0.1 \mathrm{~K}$, that are introduced in approximately the lowest $3 \mathrm{~km}$ of the model.

The topography for the simulations is based on a cross section of the Sierra Nevada terrain near the latitude of 


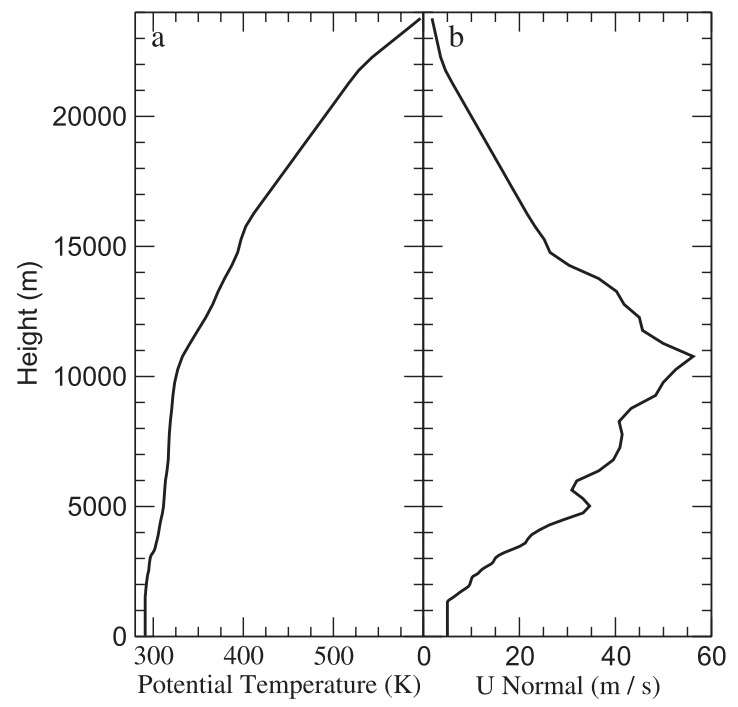

FIG. 2. Mobile GPS Advanced Upper-Air Sounding System (MGAUS) sounding at 2100 UTC 16 Apr used to display the (a) potential temperature $(\mathrm{K})$ and (b) the cross-mountain wind component $\left(\mathrm{m} \mathrm{s}^{-1}\right)$.

Independence, California. The Sierra Nevada range is asymmetric, with the lee slopes steeper than the upwind slopes. The model represents the Sierra Nevada crest mountain height as approximately $3500 \mathrm{~m}$ with the downstream Inyo Range crest height $2500 \mathrm{~m}$. The terrain upstream of the Sierra foothills and downstream of the Inyos is held constant (e.g., Fig. 15) to isolate the flow response to the topography immediately upwind and downwind of Owens Valley.

Horizontally nested grids are utilized in the simulations so as to achieve adequate resolution to explicitly resolve the internal structure of the rotors. The simulations use an infinite 2D ridge (in the $y$ direction) and have three grid meshes with horizontal resolutions of 540,180 , and $60 \mathrm{~m}$ corresponding to horizontal dimensions of $601 \times 25,241 \times 55$, and $397 \times 139$ grid points on each mesh, respectively. The simulations make use of 110 vertical levels with a vertical grid spacing that is a constant $50 \mathrm{~m}$ between the surface and $2500 \mathrm{~m}$ and then gradually stretches to a maximum spacing of $500 \mathrm{~m}$ that remains constant to the model top near $24 \mathrm{~km}$. The lowest model computational level is at $25 \mathrm{~m}$.

\section{Observations of subrotor-scale structure}

One of the strongest mountain wave events during T-REX took place on 16 April 2006 (IOP 13) and was precipitated by synoptic-scale winds-in excess of $20 \mathrm{~m} \mathrm{~s}^{-1}$ at mountain crest level-that passed over the Sierra Nevada range. The $500-\mathrm{hPa}$ geopotential height and winds valid at 1200 UTC 16 April and 0000 UTC
17 April 2006, which are based on a COAMPS analysis and 12-h simulation using a $13.5-\mathrm{km}$ horizontal grid increment, are shown in Fig. 3. A large-scale trough was positioned along the U.S. West Coast, associated with a midtropospheric wind maximum in excess of $30 \mathrm{~m} \mathrm{~s}^{-1}$ from a southwesterly direction impinging on the Sierra Nevada. As the trough amplified during 16 April, the strongest westerlies passed over the central portion of the Sierras. The strong cross-mountain flow in concert with a frontal system, characterized by enhanced lowertropospheric static stability, resulted in favorable conditions for mountain waves and associated phenomena (e.g., Colson 1954; Durran 1990).

In the afternoon hours of 16 April 2006, largeamplitude mountain waves were observed by two research aircraft in the lee of the Sierra Nevada at altitudes ranging from just above the crest through the lower stratosphere. Measurements from the University of Wyoming King Air research aircraft at altitudes below $8 \mathrm{~km}$ MSL indicate a trapped gravity wave that spanned Owens Valley with a dominant wavelength $\sim 30-35 \mathrm{~km}$, which facilitated boundary layer separation and rotor formation in the lee of the Sierra Nevada. This relatively long wavelength may be a manifestation of the topography surrounding Owens Valley. For example, the idealized two-dimensional simulations of Grubišić and Stiperski (2009) of flow over a double-bell-shaped obstacle indicate that two ridges with a separation distance corresponding to Owens Valley generate trapped lee waves with a wavelength that is about $20 \%$ longer than for waves generated by a single ridge. Although a cloud and precipitation shield existed upstream of the Sierra, in general there were very few clouds in the lee during this event. However, a water vapor image from the Moderate Resolution Imaging Spectroradiometer (MODIS) valid at 2015 UTC 16 April 2006 exhibits a pattern of dry and moist lineal features consistent with trapped gravity waves in the lee of the Sierra Nevada (not shown). The horizontal wavelength is longer in the central Sierra region and shorter to the south, likely a reflection of the weaker cross-mountain wind speed to the south and a direction that is not perpendicular to the ridge line, in contrast to the region farther north and upstream of Independence.

Strong downslope flow in excess of $20 \mathrm{~m} \mathrm{~s}^{-1}$ was observed by the T-REX surface stations in the upper parts of the Sierra Nevada lee slopes. During this time, the DRI surface observational network within Owens Valley, which is arranged in three cross-valley lines that extend from the valley floor to the alluvial fan at the base of the Sierra Nevada lee slopes (Fig. 1), shows intermittent periods (5-15 min in length) of variable easterly winds over the upper parts of the alluvial slope at the western 

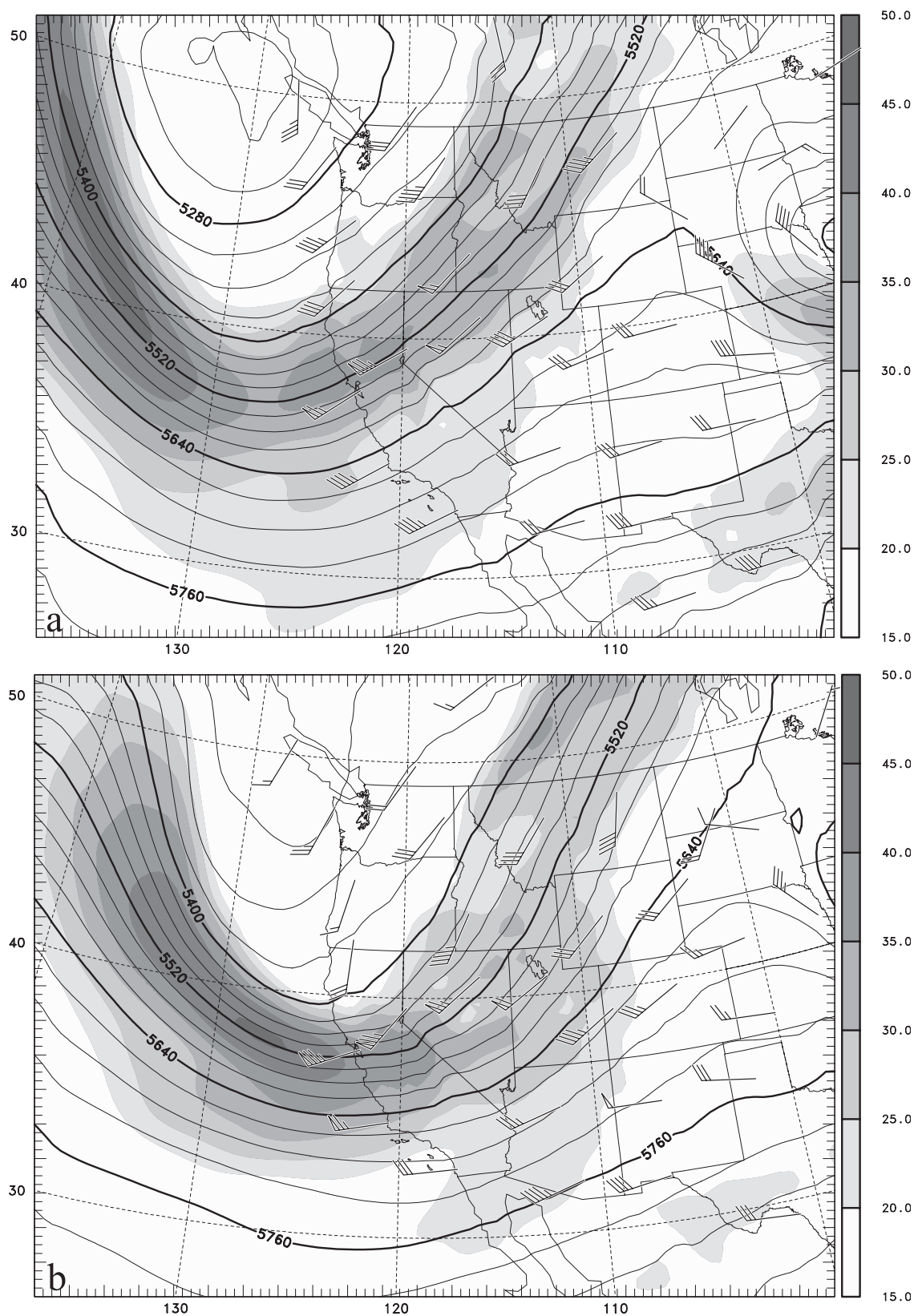

FIG. 3. The 500-hPa geopotential height and wind speed at (a) 1200 UTC 16 Apr 2006 (analysis) and (b) 0000 UTC 17 Apr 2006 (12-h forecast) from COAMPS using a 13.5-km resolution. Radiosonde wind observations are shown in (a) with a full wind barb corresponding to $5 \mathrm{~m} \mathrm{~s}^{-1}$ and a pennant representing $25 \mathrm{~m} \mathrm{~s}^{-1}$. The geopotential height is shown every $30 \mathrm{~m}$, with wind speed in gray shading increments of $5 \mathrm{~m} \mathrm{~s}^{-1}$ above $20 \mathrm{~m} \mathrm{~s}^{-1}$.

edge of the network at elevations between $\sim 1500$ and $1700 \mathrm{~m}$ MSL, as shown in Fig. 4. Farther down the slope, in the central portion of the valley, strong south to southwesterly winds were documented by the surface network during the afternoon hours of 16 April. The strong westerlies penetrated down the slopes to the western portion of the DRI network by 2300 UTC
(Fig. 4a), coincident with an abrupt change in the mean surface flow in the central portion of the valley from a southerly to more of a westerly and northwesterly direction (Fig. 4b). An adverse low-level pressure gradient of $\sim 0.03 \mathrm{hPa} \mathrm{km}{ }^{-1}$ during the 1900-2200 UTC 16 April period is present across the axis of the valley, as apparent in the pressure perturbation time series 

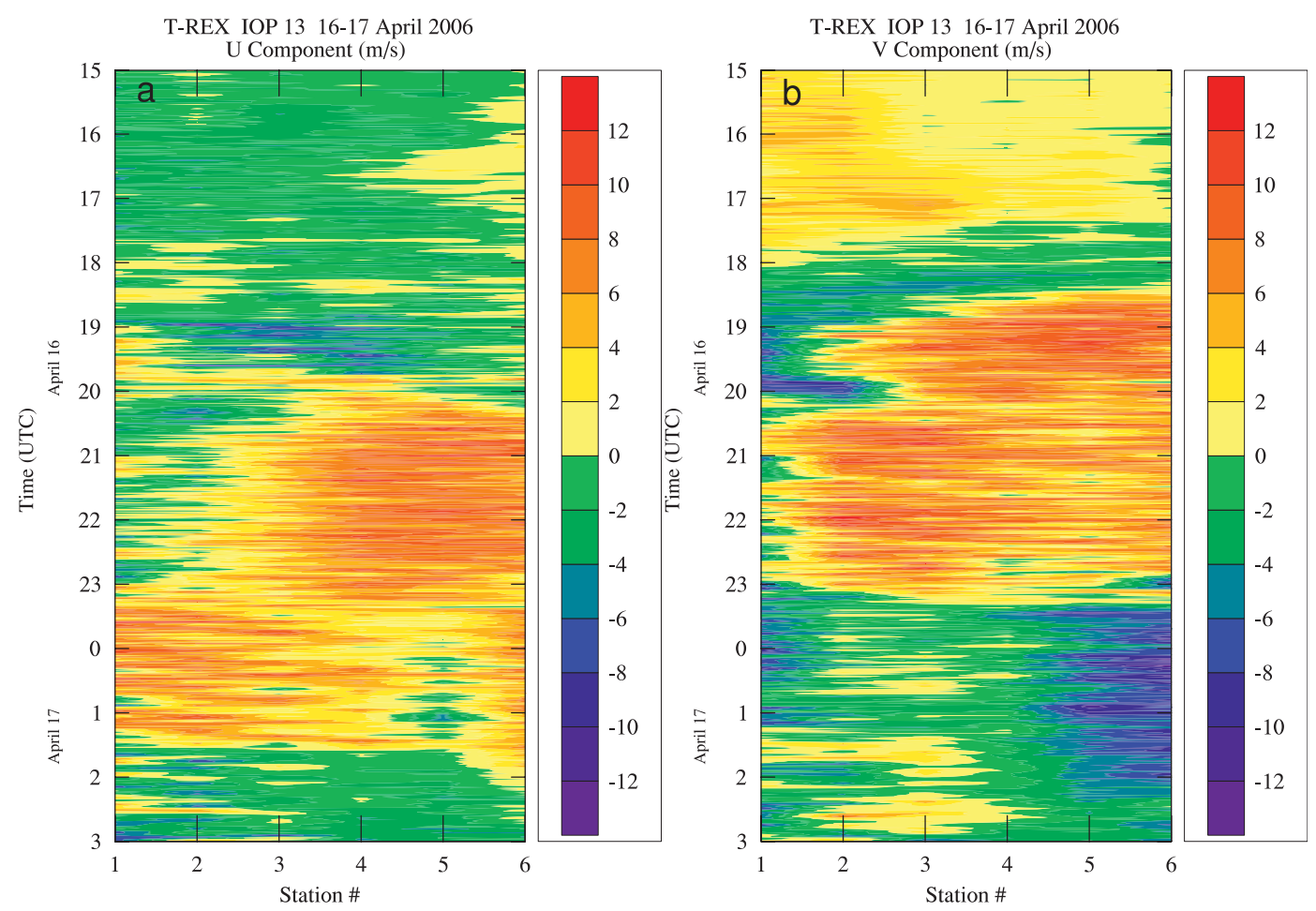

FIG. 4. Hovmöller diagram showing the temporal evolution from 1500 UTC 16 Apr to 0300 UTC 17 Apr 2006 for the (a) $u$ and (b) $v$ wind components derived from DRI surface network stations $1-6$, which span the base of Owens Valley. The wind speeds are color shaded using $2 \mathrm{~m} \mathrm{~s}^{-1}$ increments.

shown in Fig. 5. The adverse pressure gradient may be induced by the trapped lee waves aloft. There are periods when the adverse pressure gradient relaxes or even reverses, such as are apparent in the 0000-0100 UTC 17 April time period. High-frequency oscillations are present in the pressure perturbation time series and exhibit a rapid eastward propagation across the valley, particularly during the 2000-2100 UTC 16 April time period. The pressure perturbation was computed based on the 30-s dataset and filtered to remove the diurnal and semidiurnal tidal signatures.

The DLR high-resolution Doppler lidar was deployed to provide quantitative observations of the internal structure of mountain waves and rotors. Conical plan position indicator (PPI) and vertical range height indicator (RHI) scans were performed in a coordinated manner throughout the 16-17 April time period. A conical PPI scan at an elevation of $18^{\circ}$ valid at 2154 UTC 16 April 2006 is shown in Fig. 6. The conical scan shows strong westerly downslope flow (directed toward the lidar) in excess of $20 \mathrm{~m} \mathrm{~s}^{-1}$ (indicated by the dark blue colors in Fig. 6) along the lee slopes that rapidly transitions to localized regions of reversed flow (directed away from the lidar or easterly in this case) at altitudes of $0.5-2 \mathrm{~km}$ AGL. At an elevation angle of $3^{\circ}$ valid at $2150 \mathrm{UTC}$, the strong southerly flow apparent near the surface is consistent with the DRI surface network at this time. At higher altitudes the strong southwesterly flow nearly perpendicular to the Sierra crest is evident.

A sequence of RHI radial velocity scans along a vertical slice derived from the Doppler lidar for 2130 2135 UTC 16 April 2006 is shown in Figs. 7a-d. The strong downslope flow is once again apparent along the leeward slopes of the eastern Sierra Nevada. At a distance of $10 \mathrm{~km}$ to the west of the lidar, the most intense downslope wind velocities are located just above the surface. Farther downstream along the lee slopes, $5 \mathrm{~km}$ to the west of the lidar, the strongest flow is elevated $1-2 \mathrm{~km}$ above the surface, consistent with boundary layer separation from the surface induced by adverse pressure gradients. Beneath the strong winds aloft, the lidar indicates the presence of localized regions along the western side of the valley where the flow is directed away from the lidar and back toward the Sierra slopes, suggestive of the presence of small-scale vortices or subrotors. The radial wind component directed back toward the Sierras is consistent with the low-level southeasterly flow. Near a region of large vertical wind shear at approximately $3 \mathrm{~km}$ MSL and $2 \mathrm{~km}$ upstream of the lidar, 


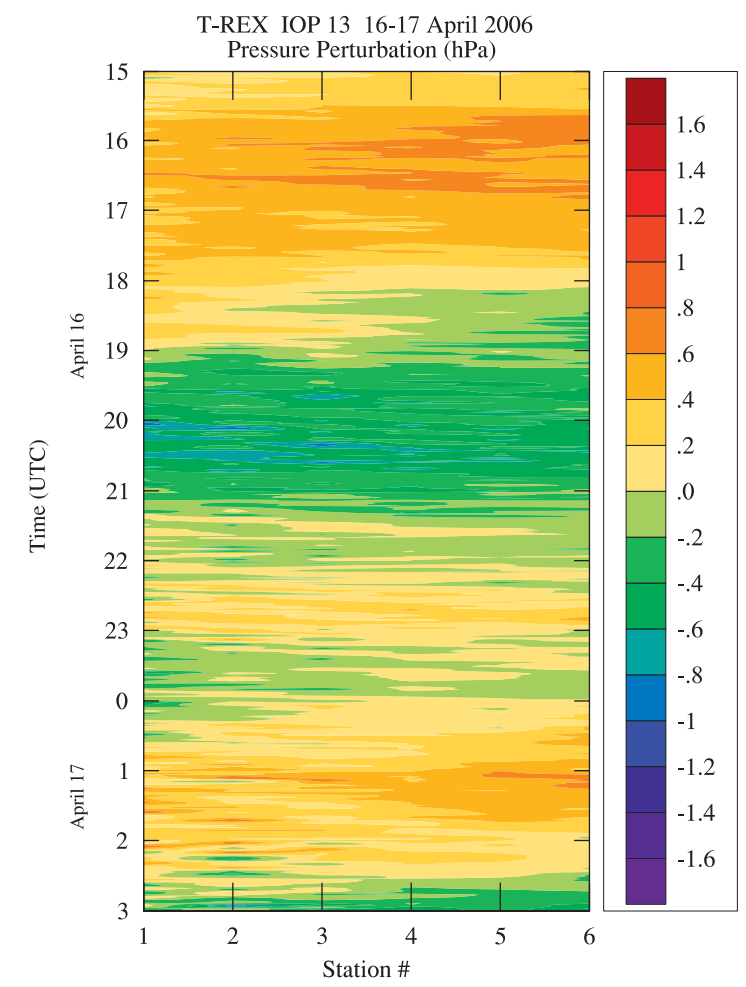

FIG. 5. Surface perturbation pressure Hovmöller diagram for the 1500 UTC 16 Apr-0300 UTC 17 Apr 2006 time period derived from DRI surface network stations 1-6, which span the base of Owens Valley. The pressure perturbations are color shaded using $0.2 \mathrm{hPa}$ increments.

a vortex develops at the shear interface. The subrotor vortex intensifies over the 5 -min time period shown in Fig. 7 and attains a distinct small-scale circulation that is apparent in the radial velocity by 2135 UTC (Fig. 7d). The scale of this circulation is approximately $1 \mathrm{~km}$.

One of the major challenges for T-REX was not only to confirm the existence of subrotor vortices but also to provide an estimate of the vorticity within the subrotor circulations. The shear in the small-scale circulation shown in Fig. $7 \mathrm{~d}$ exceeds $35 \mathrm{~m} \mathrm{~s}^{-1}(200 \mathrm{~m})^{-1}$. It follows that the $y$ component of the horizontal vorticity, $\eta=(\partial u / \partial z-\partial w / \partial x)$, should be large. The $\eta$-vorticity component can be obtained through computation of the tangential velocity component derived using continuity in the $x-z$ plane following Weissmann et al. (2009). In polar coordinates, the continuity equation for a twodimensional incompressible flow can be written as

$$
\frac{1}{r} \frac{\partial}{\partial r}\left(r V_{r}\right)+\frac{1}{r} \frac{\partial V_{\theta}}{\partial \theta}=0
$$

where $\left(V_{r}, V_{\theta}\right)$ denote the radial and tangential components of the velocity, respectively. A boundary con-

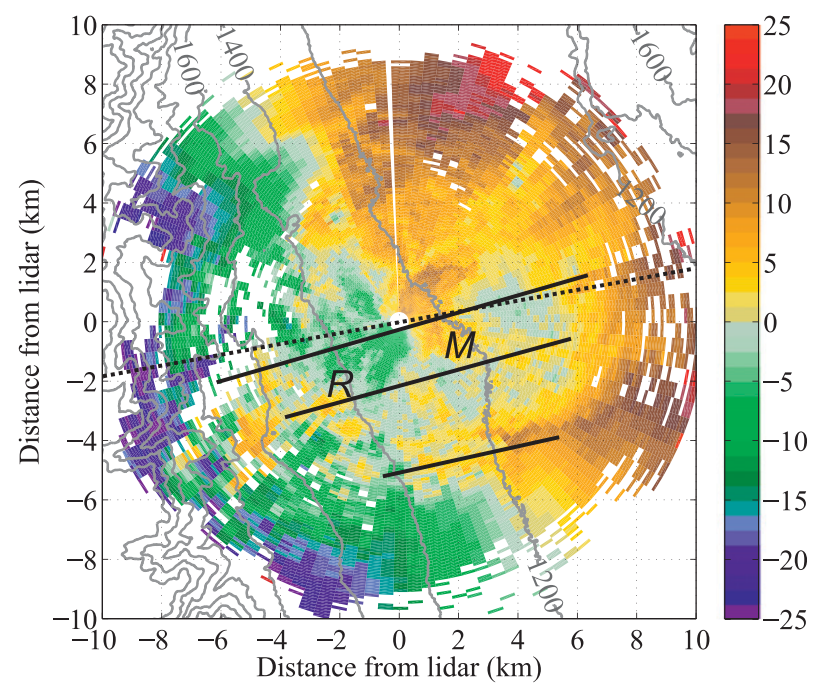

FIG. 6. Doppler lidar radial velocity $\left(\mathrm{m} \mathrm{s}^{-1}\right)$ for a conical scan with $18^{\circ}$ elevation at 2154 UTC 16 Apr. Warm colors (yellow and red) indicate flow away from the lidar and cold colors (green and blue) correspond to flow toward the lidar. Locations of the REAL (R) and MAPR (M) instruments are shown, with the axes showing horizontal distance $(\mathrm{km})$. Topography $(\mathrm{m})$ is shown by the gray contours. The azimuth is $80^{\circ}$ for the vertical scan, shown by the dotted line segment. The solid lines show the approximate location of the three lines of the DRI surface station network (16 units).

dition of zero tangential velocity (i.e., $V_{\theta}=0$ ) is assumed at the surface and the tangential velocity is calculated incrementally for every quadrant. The $y$-vorticity component in polar coordinates can then be defined as

$$
\eta=\frac{1}{r} \frac{\partial}{\partial r}\left(r V_{\theta}\right)-\frac{1}{r} \frac{\partial V_{r}}{\partial \theta} .
$$

Additionally, an along-beam smoothing of the calculated tangential component with the neighboring range gates is applied, which reduces errors associated with convergence perpendicular to the $x-z$ plane, as revealed in comparisons with model simulations (Weissmann et al. 2009). Application of this method to vorticity derived from model simulated fields indicates that the derived vorticity can explain $66 \%$ of the mean square vorticity fluctuations and has a reasonable magnitude.

The calculation of the $y$-vorticity component corresponding to the 2135 UTC 16 April radial scan is shown in Fig. 8. The subrotor circulation that is apparent in Fig. $7 \mathrm{~d}$ corresponds to a horizontal vorticity maximum of $\sim 0.2 \mathrm{~s}^{-1}$. After this particular subrotor develops, it advects out of the plane of the lidar scan as it weakens. Multiple vortex signatures are apparent in the lidar vertical scans, particularly during the 2000-2300 UTC 

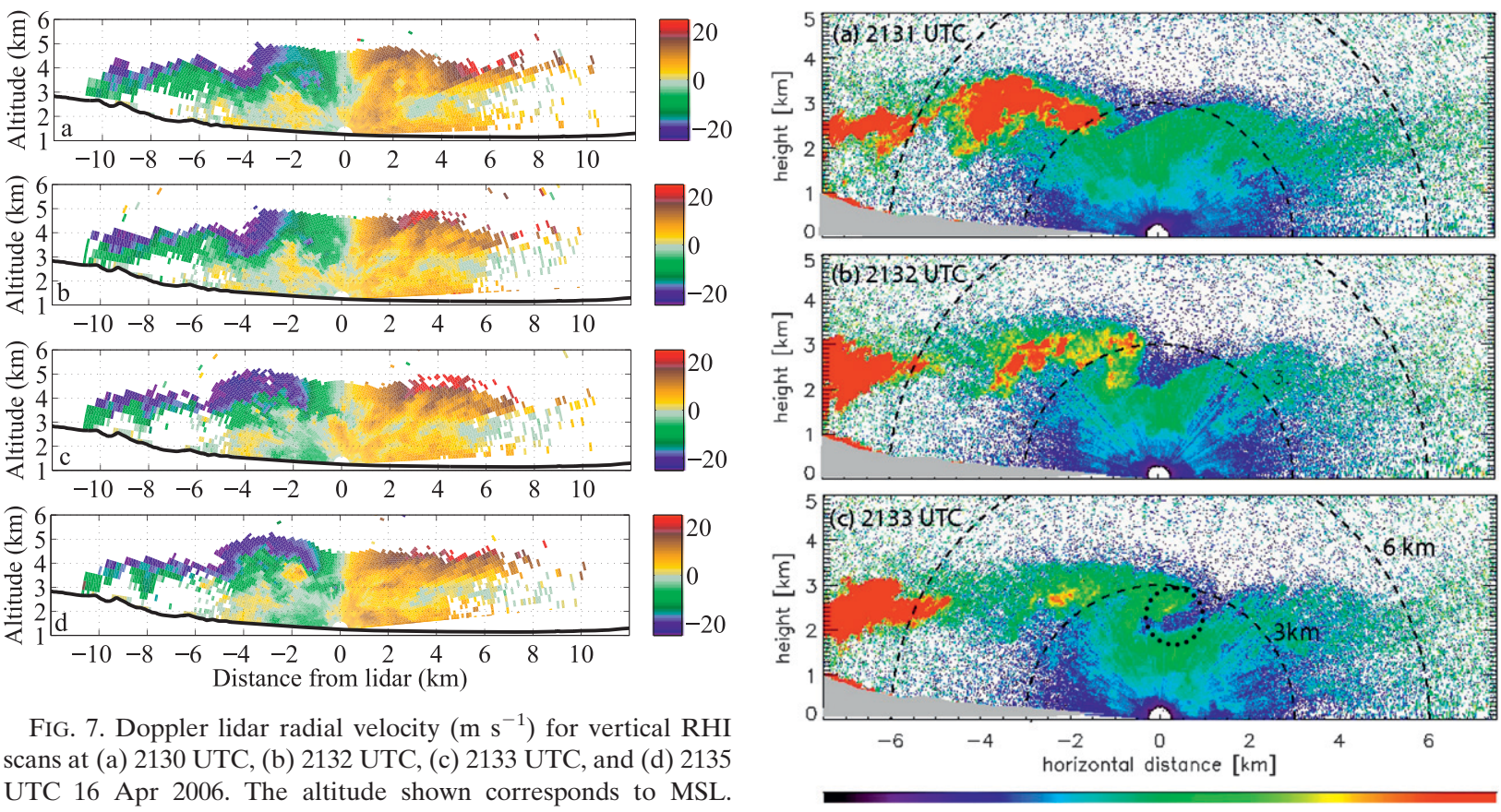

FIG. 7. Doppler lidar radial velocity $\left(\mathrm{m} \mathrm{s}^{-1}\right)$ for vertical RHI scans at (a) 2130 UTC, (b) 2132 UTC, (c) 2133 UTC, and (d) 2135 UTC 16 Apr 2006. The altitude shown corresponds to MSL. Vertical scans are oriented across Owens Valley nearly perpendicular to the Sierra crest with a west-east configuration corresponding to left-right in the figure. The mean downslope flow is from left to right. The color convention is as in Fig. 6.

time period. For example, RHI radial scans for 2142 and 2144 UTC 16 April (not shown) once again indicate the development of a billow along the shear interface approximately $2-4 \mathrm{~km}$ to the west of the lidar.

Aerosol backscatter measurements from NCAR REAL at 1-min intervals between 2131 and 2133 UTC 16 April 2006 (along an $80^{\circ}$ azimuth), shown in Fig. 9, indicate that plumes of aerosols and a few clouds are advected along the lee slopes prior to flow separation and are lofted and advected downstream in a layer of elevated strong downslope flow, consistent with the Doppler lidar observations of the subrotor (Fig. 7). The motion implied by the aerosol backscatter fields shown in Figs. 9a-c is consistent with a vortex circulation embedded within the rotor in the plane of scan, as also

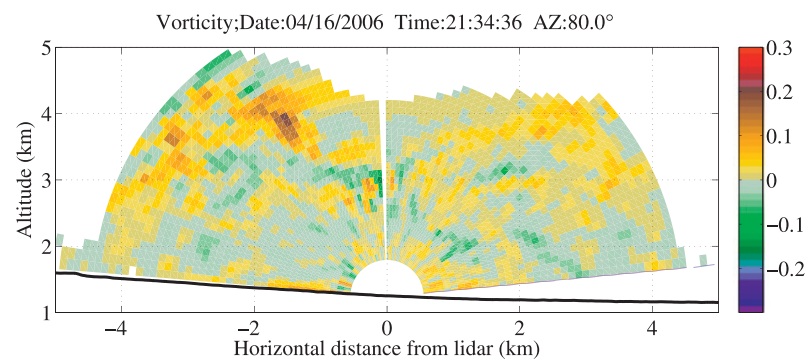

FIG. 8. Estimate of the $y$-vorticity component $\left(\mathrm{s}^{-1}\right)$ based on the DLR lidar radial velocity for a vertical RHI scan at 2135 UTC 16 Apr 2006.

Backscatter ratio (-)

highest

FIG. 9. Aerosol backscatter from the REAL system for (a) 2131 UTC, (b) 2132 UTC, and (c) 2133 UTC 16 Apr 2006. The scan is oriented across the Owens Valley nearly perpendicular to the Sierra crest with a west-east configuration corresponding to a leftright orientation in the figure. Range rings are plotted every $3 \mathrm{~km}$. The mean downslope flow is from left to right. The dotted circle in (c) highlights the subrotor circulation.

evident in the Doppler lidar observations. The upper portion of the aerosol layer overturns and folds over on itself as a result of the vortex amplification and associated circulation. This sequence of aerosol scans suggests that subrotors may be important for entrainment and detrainment along the boundary layer top and may provide a mechanism for the aerosols within the boundary layer to be ejected into the free atmosphere and transported downstream. These finescale structures appeared frequently during the 2100 UTC 16 April-0230 UTC 17 April period, as deduced by timeheight analysis of aerosol backscatter. For example, a Hovmöller diagram of the aerosol backscatter ratio along the top of the aerosol layer derived from REAL for the 0125-0225 UTC time period is shown in Fig. 10. The top of the aerosol layer is chosen based on the vertical gradient of the backscatter along the scan. Numerous high-backscatter ratio events are apparent, which may contain vortices similar to that observed in the 2130-2135 UTC time period (Fig. 9). The time series indicates a rapid progression eastward of the enhanced backscatter events at a $13 \mathrm{~m} \mathrm{~s}^{-1}$ velocity, broadly similar to the advective velocity in the $3-4-\mathrm{km}$ layer as deduced 


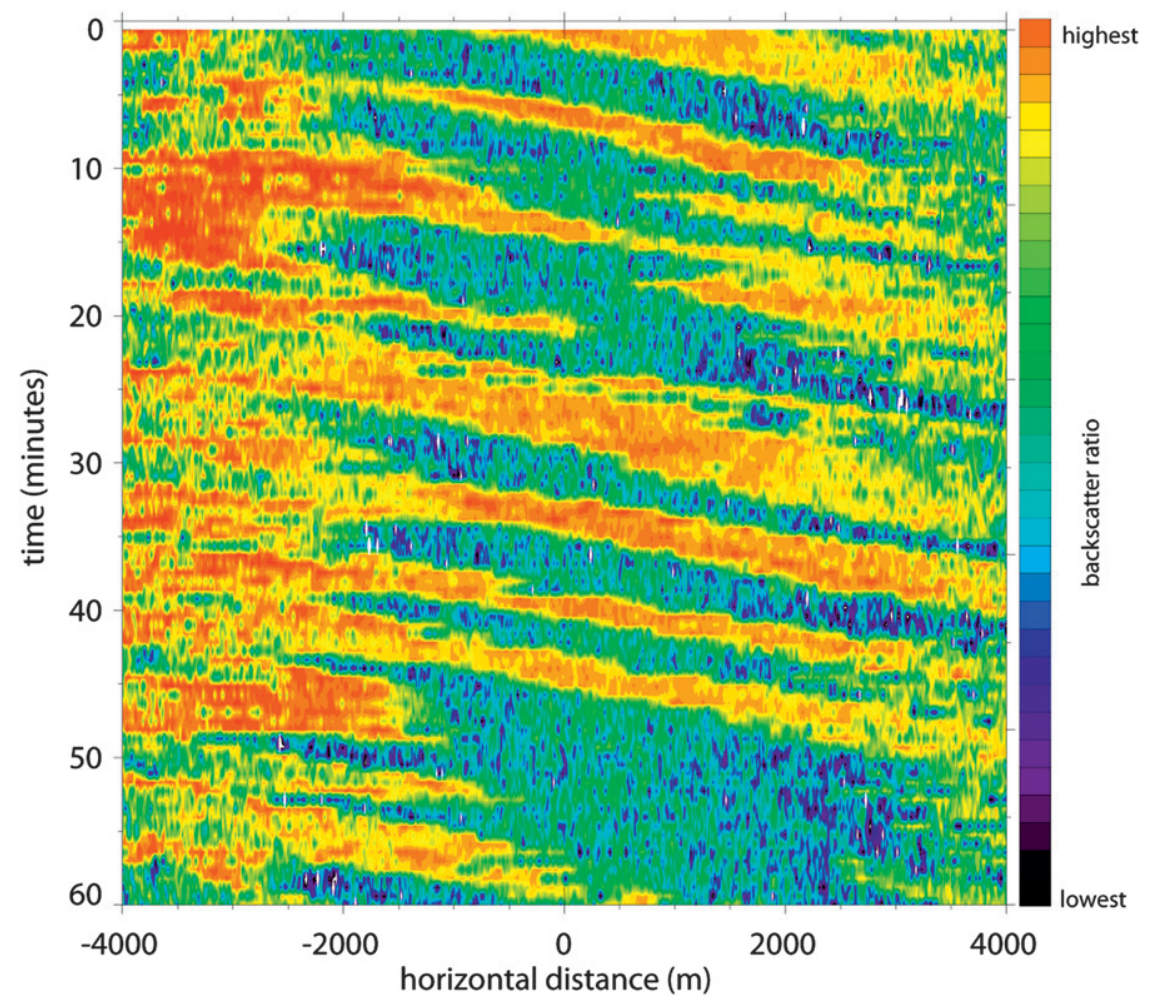

FIG. 10. Hovmöller diagram of the aerosol backscatter ratio along the top of the aerosol layer derived from REAL for the 0125-0225 UTC time period. Warmer colors represent larger backscatter ratios.

from radiosonde ascents launched in the valley on the afternoon of 16 April.

The NCAR MAPR also confirmed the presence of high-frequency, small-scale coherent structures, as illustrated in Fig. 11. During 2115-2315 UTC, the MAPR measured a series of updrafts and downdrafts that passed over the profiler at a frequency of $\sim 7 \mathrm{~h}^{-1}$ (Fig. 11a). The vertical velocity maxima and minima appear to be a signature of strong vertical mixing and are also associated with regions of enhanced localized cross-valley westerly flow, punctuated by periods of reversed flow directed back toward the Sierra crest in the $1.5-2.5-\mathrm{km}$ AGL layer (see Fig. 14b). These "fossil" subrotor vortices likely formed over the western portion of the valley along the shear zone associated with the leading edge of the rotor and subsequently advected downstream over the remote sensing platforms, including the MAPR. The King Air in situ aircraft data indicate that the ascending branch of the lee wave is located along the western portion of the valley during the afternoon hours of 16 April (not shown), lending further support to the fossil subrotor interpretation. The relatively high reflectivity shown in Fig. 11c suggests sharp gradients in refractivity (a function of humidity and temperature) and is indicative of the dynamic activity during this time period. Figures 11a,b imply localized positive maxima of $y$ vorticity associated with subrotors near the top of the turbulence layer. The spectral Doppler width maximum in the 2.5-3.5-km layer (Fig. 11d) indicates the presence of moderate turbulence associated with these subrotor vortices. Observations from the REAL and Doppler lidars, in addition to the MAPR, all exhibit frequent occurrences of small-scale turbulent eddies and vortices in the 1.5-2.5-km AGL layer during this case.

The pressure perturbation power spectrum from DRI surface station tower 4 (Fig. 1), located near the MAPR site, based on the 0800 UTC 16 April-0800 UTC 17 April time period is shown in Fig. 12. The spectra shown in Fig. 12 are computed using the 30-s pressure dataset from tower 4 using Fourier transforms following the technique outlined in Cuxart et al. (2002). A maximum in the power spectrum on 16-17 April is apparent for frequencies in the $6-12 \mathrm{~h}^{-1}$ range, reasonably consistent with the observations from MAPR and the REAL time series. It should be noted that the spectrum at surface station tower 1 (not shown) located at the western edge of the DRI surface network, exhibits generally similar characteristics as for tower 4 , including several distinct high-frequency maxima. In contrast, the power spectrum for tower 4 on the day prior (15-16 April) 


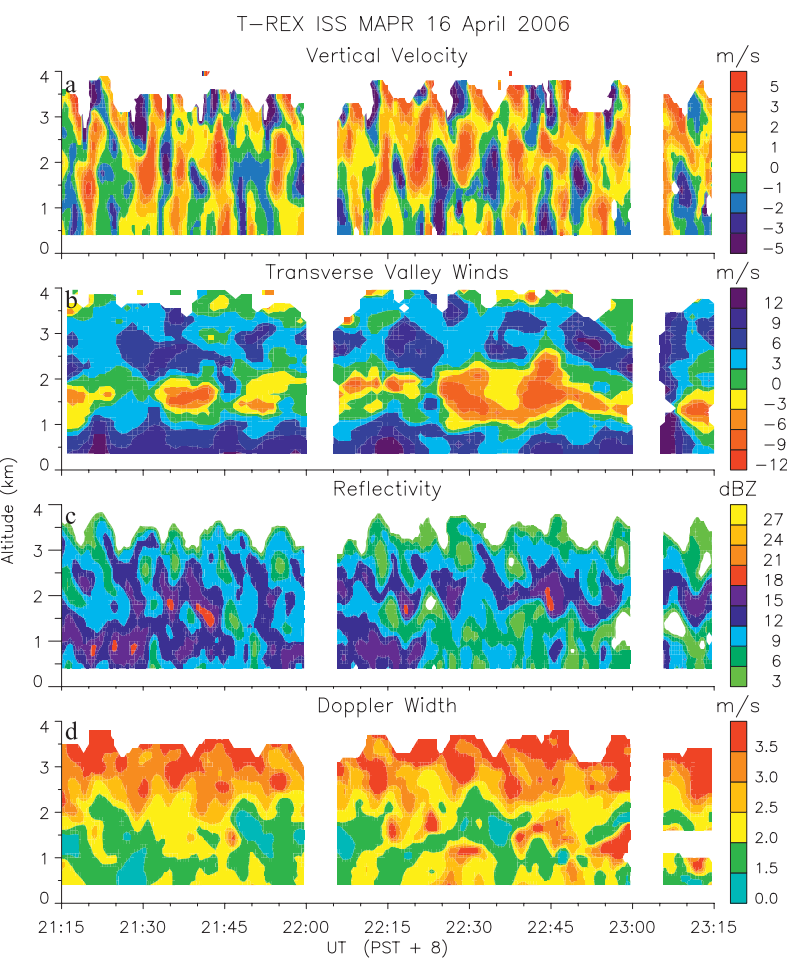

FIG. 11. MAPR wind profiler observations of (a) vertical velocity $\left(\mathrm{m} \mathrm{s}^{-1}\right),(\mathrm{b})$ transverse-valley wind component $\left(\mathrm{m} \mathrm{s}^{-1}\right)$, (c) reflectivity (dBZ), and (d) Doppler width $\left(\mathrm{m} \mathrm{s}^{-1}\right)$ for 2115-2315 UTC 16 Apr 2006. Negative values of the transverse-valley wind component correspond to a wind direction oriented toward the Sierras. The altitude shown is AGL.

indicates less energy in the higher frequencies (Fig. 12), a reflection of the more quiescent conditions that featured trapped waves with no rotors present and less turbulent conditions. The spectra in general follow a $-5 / 3$ slope and are consistent with inertial subrange scaling, similar to that found in other boundary layer observations.

The high-frequency character of the turbulent flow is further illustrated in Fig. 13a, which shows the vertical velocity measured by the King Air for a flight leg $14.6 \mathrm{~km}$ in length that extends across Owens Valley during 2354-2357 UTC 16 April at $2450 \mathrm{~m}$ MSL. A series of updrafts and downdrafts are evident in the vertical velocity observations. A wavelet power spectrum analysis of the vertical velocity ( $25-\mathrm{Hz}$ aircraft measurements), shown in Fig. 13b, indicates a maximum in the power spectrum near a period of $12 \mathrm{~s}$, or approximately $1-\mathrm{km}$ wavelength. This maximum was observed over a period of $\sim 70 \mathrm{~s}$, or a horizontal distance of $\sim 7 \mathrm{~km}$. Power spectra derived from the aircraft observations at other altitudes below $4 \mathrm{~km}$ indicate similar characteristics of the turbulence. The cospectrum of the vertical velocity and potential temperature perturbation is characterized by a negative maximum in the period near $12 \mathrm{~s}$ (not

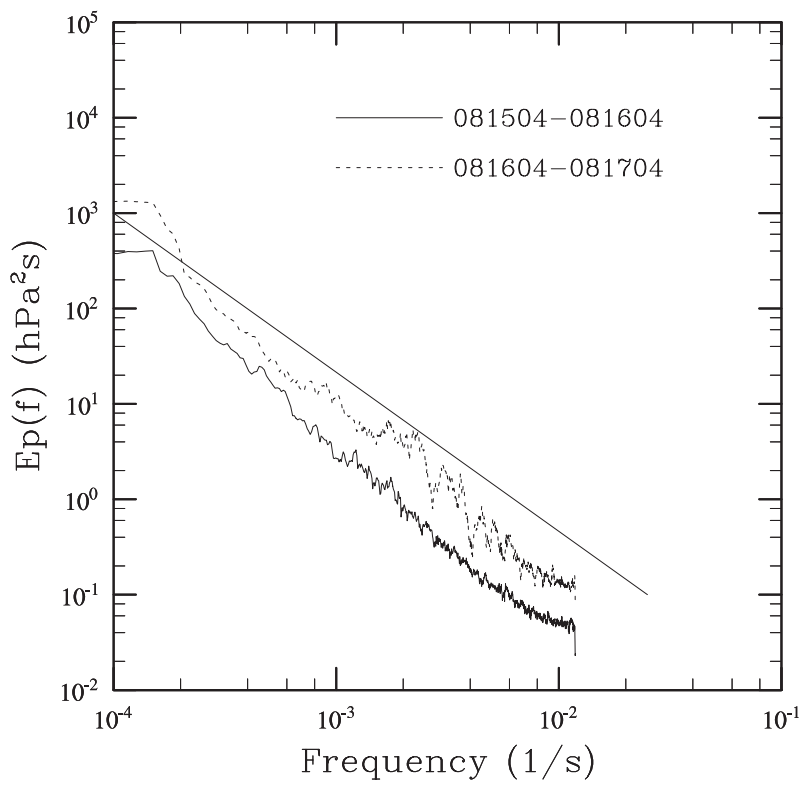

FIG. 12. Power spectrum of surface pressure perturbations based on the data from DRI surface station tower number 4 located near the MAPR site for 0800 UTC Apr 15 through 0800 UTC Apr 16 (solid) and 0800 UTC Apr 16 through 0800 UTC Apr 17 (dashed). The $-5 / 3$ slope is shown by the thin straight line.

shown). The negative sensible heat flux, that is, downward heat transfer, indicates convective stability, suggestive that these large eddies were likely shear generated. The shear generation mechanism is consistent with the Richardson number profile shown in Fig. 14d, which is nonnegative and less than unity near $2.5 \mathrm{~km}$ MSL. These observations of coherent turbulent eddies with horizontal scales of approximately $1 \mathrm{~km}$ are consistent with those obtained from the ground-based remote sensing platforms discussed previously.

A radiosonde ascent from Owens Valley at 2100 UTC, shown in Fig. 14, indicates strong vertical wind shear, approximately $20 \mathrm{~m} \mathrm{~s}^{-1} \mathrm{~km}^{-1}$ in the $3.0-4.5-\mathrm{km}$ layer MSL (1.5-3.0 km AGL) (Fig. 14b), which corresponds to the layer where the subrotor was observed by the Doppler lidar and the other ground-based instrumentation. The strong wind shear was coupled with a layer of relatively weak static stability (Fig. 14a) that results in a reduction of the dimensionless gradient Richardson number, $\mathrm{Ri}$, to less than 0.25 and in some layers less than 0.0 (Fig. 14d), indicative of turbulence generation and convective overturning. The Ri profile is computed based on smoothed layer-averaged wind and potential temperature profiles from the sounding. The layer of subcritical $\mathrm{Ri}$ points to the potential for a Kelvin-Helmholtz-like instability for subrotors similar to that found in highresolution numerical simulations (Klaassen and Peltier 1985; Fritts et al. 1996; Doyle and Durran 2007). 


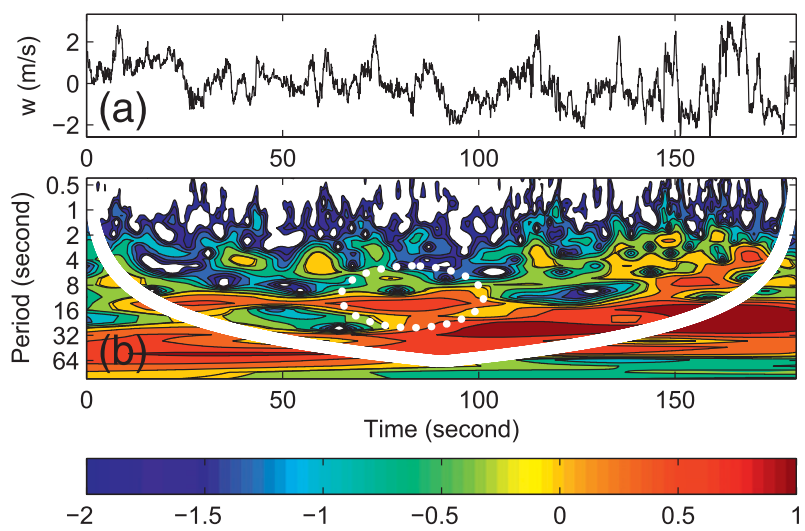

FIG. 13. (a) Vertical velocity derived from a King Air flight leg at 2450-m altitude corresponding to 2354-2357 UTC 16 Apr. (b) The wavelet power spectrum for vertical velocity (shaded). The $95 \%$ statistical confidence level is shown by the white solid curve. The length of the flight leg is $14.6 \mathrm{~km}$. The log of the power spectrum is shown and shaded in color. The power spectrum maximum of particular interest is highlighted by the white dotted oval

\section{Numerical simulations}

\section{a. Simulation of IOP 13}

High-resolution three-dimensional eddy resolving simulations were conducted using a triple- nested grid configuration of COAMPS and initialized with a vertical profile derived from a radiosonde ascent upstream of the mountains at 2100 UTC 16 April 2007 (Fig. 2). A vertical section of potential temperature and vertical velocity for the 540-m resolution outermost grid mesh, which is displayed for the central axis and normal to the Sierra crest, is shown in Fig. 15. A series of trapped mountain waves are apparent in the lee of the Sierra and Inyo mountains. A single wave crest is present over Owens Valley, very similar to the UW King Air research aircraft measurements of potential temperature (not shown).

The $y$ component of the horizontal vorticity (spanwise) for the $60-\mathrm{m}$ resolution mesh at $3.5 \mathrm{~km}$ MSL for the 3.5-h and 4.0-h simulation times is shown in Fig. 16. The high-resolution mesh results indicate the presence of a series of vortices or subrotors, particularly near the region where the vortex sheet intersects with the $3.5-\mathrm{km}$ plane. Vertical sections of the horizontal vorticity, potential temperature, and $u$ wind component are shown in Fig. 17 for the 3.5- and 4.0-h simulation times along cross sections A-A' and B-B' (Fig. 16). Subrotors are evident along the ascending branch of the lee wave in the region of strong vertical wind shear (Figs. 17b,d). The subrotors attain a maximum in the horizontal vorticity of approximately $0.2 \mathrm{~s}^{-1}$, similar to the horizontal vorticity derived from the DLR lidar (Fig. 8). It appears

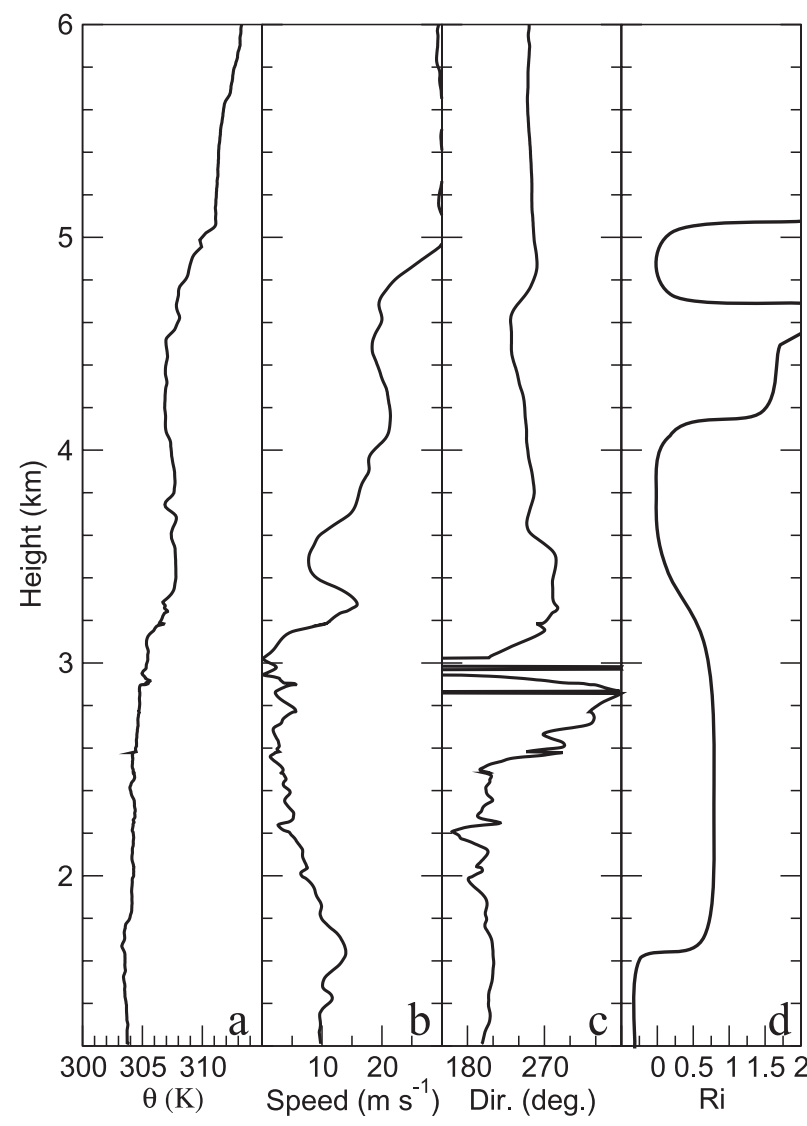

FIG. 14. Profiles derived from a radiosondes ascent at 2100 UTC 16 Apr near the MAPR profiler site corresponding to the (a) potential temperature $(\mathrm{K})$, (b) wind speed $\left(\mathrm{m} \mathrm{s}^{-1}\right)$ and (c) direction $\left.{ }^{\circ}\right)$, and (d) Richardson number.

that the model may overestimate the frequency of some of the subrotors, relative to the Doppler lidar observations, likely due to the infinite ridge in the $y$ direction that is used. The model resolution of $60 \mathrm{~m}$ may be insufficient to capture the high-frequency portion of the turbulence spectrum, which may impact the characteristics of the subrotors as well.

Time-height sections from a grid point beneath the lee wave crest (location shown in Fig. 16a) that is approximately $4 \mathrm{~km}$ downstream from the boundary layer separation point are shown in Fig. 18. The time-height section corresponds to the 3.25-3.75-h simulation times and is constructed using the model fields with a 20 -s frequency. The $u$ wind component time-height section (Fig. 18a) shows that regions of enhanced reversed flow occur episodically, with an approximate refresh rate of every 5-8 min. These regions of reversed flow are correlated with subrotor vortices that develop along the vortex sheet and advect downstream, as indicated in the $y$-horizontal vorticity time-height section shown in Fig. 18b. In general the regions of reversed flow appear to 


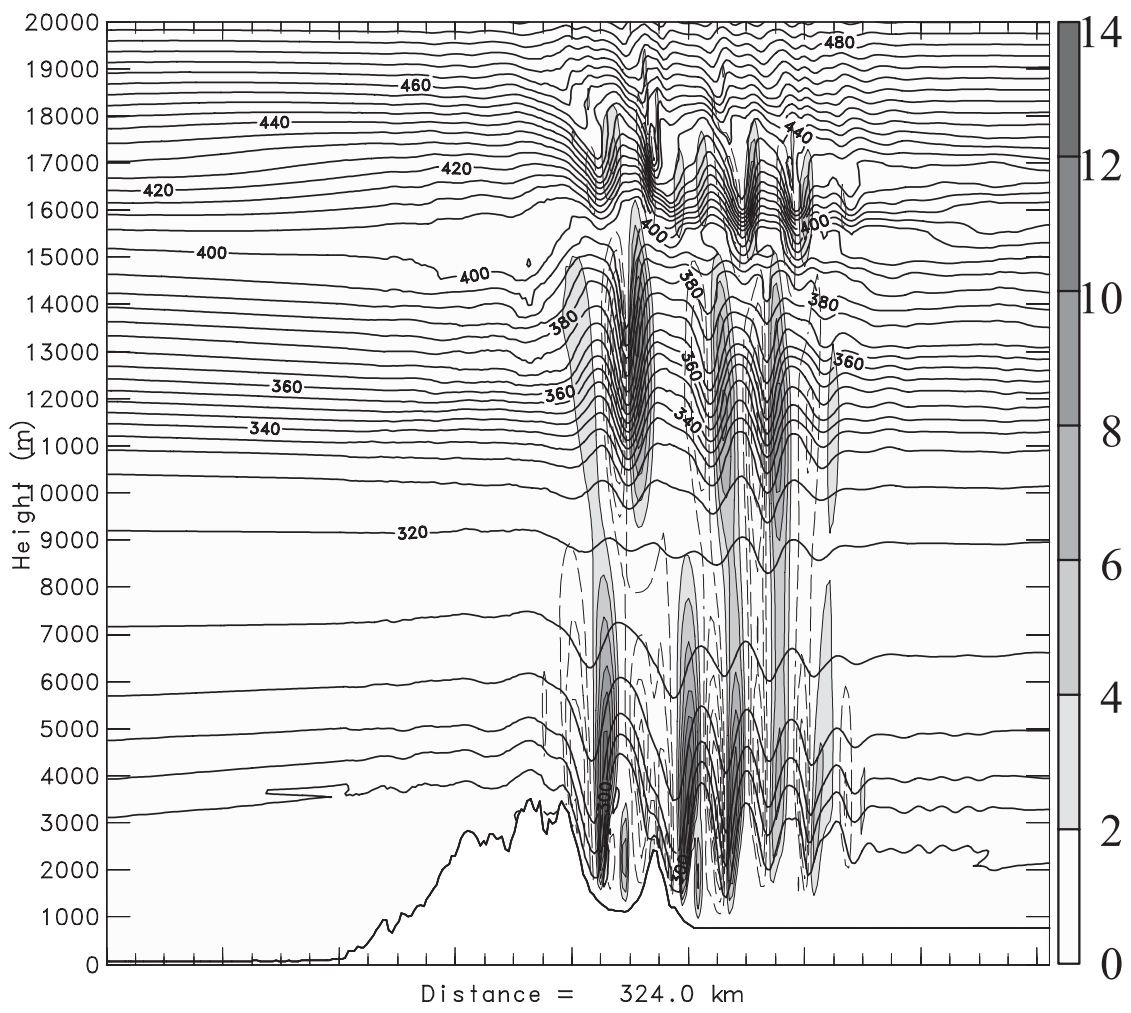

FIG. 15. Potential temperature (K) and vertical velocity $\left(\mathrm{m} \mathrm{s}^{-1}\right)$ vertical cross section along the center axis and normal to the Sierra crest for the 540-m resolution grid mesh at the $3.5-\mathrm{h}$ simulation time. The potential temperature isotherm interval is $5 \mathrm{~K}$ and the vertical velocity isotach interval is $2 \mathrm{~m} \mathrm{~s}^{-1}$, with the zero contour suppressed.

be elevated above the surface, similar to the wind profiler observations (Fig. 11b).

A three-dimensional depiction of the downslope flow, vortex sheet, and subrotor vortices is shown in Fig. 19 for the 3-h simulation time. A series of vortices forms along the vortex sheet at the interface between the weak flow beneath the lee wave and the stronger flow aloft. These vortices subsequently detach and advect downstream, creating an elevated zone of strong turbulence near the crest level of the Sierra. The vortices also contain considerable three-dimensional structure in the $y$ direction. For example, tubes of horizontal vorticity with maxima greater than $0.15 \mathrm{~s}^{-1}$ are oriented in the $y$ direction, approximately normal to the mean flow. Beneath the lee wave within the broader rotor-scale circulation, the flow is more chaotic with episodic periods of localized reversed flow directed back toward the mountains.

\section{b. Vorticity generation}

To gain insight into the dominant vortex generation mechanism for the development of the subrotors and vorticity structure, a vorticity budget is computed. The tendency equation for the $y$-component horizontal vorticity

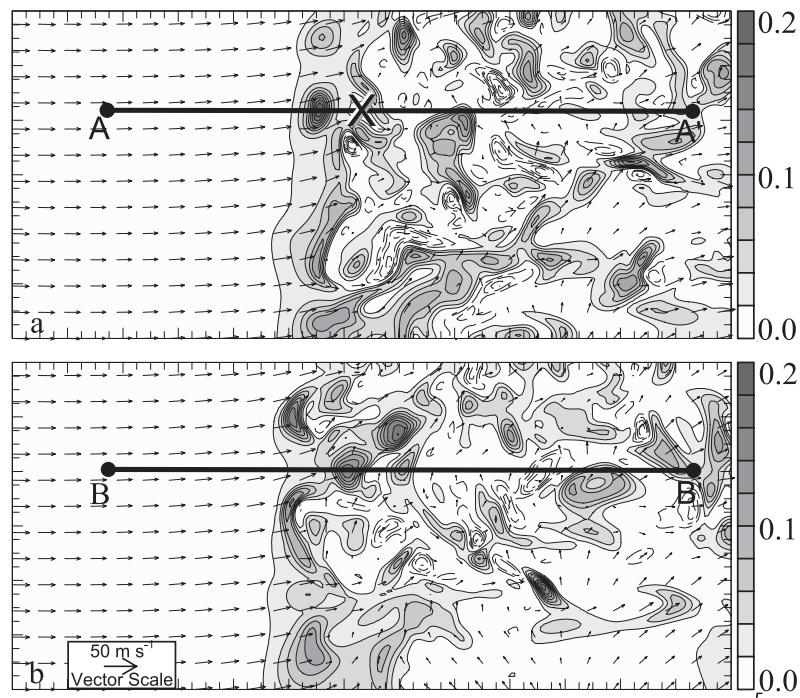

FIG. 16. Horizontal vorticity $\left(\mathrm{s}^{-1}\right) y$ component and wind vectors at $3.5 \mathrm{~km}$ (MSL) for the $60-\mathrm{m}$ resolution grid mesh at the (a) $3.5-\mathrm{h}$ and (b) 4.0-h simulation times. The horizontal vorticity shading interval is $0.01 \mathrm{~s}^{-1}$. The locations of cross sections $\mathrm{A}-\mathrm{A}^{\prime}$ and $\mathrm{B}-\mathrm{B}^{\prime}$ are shown as well. A subdomain of the 60-m-resolution mesh is shown with minor tick marks shown every $250 \mathrm{~m}$ and major tick marks every $1 \mathrm{~km}$. The location of the time-height section shown in Fig. 18 is denoted by the $\times$ symbol in (a). 

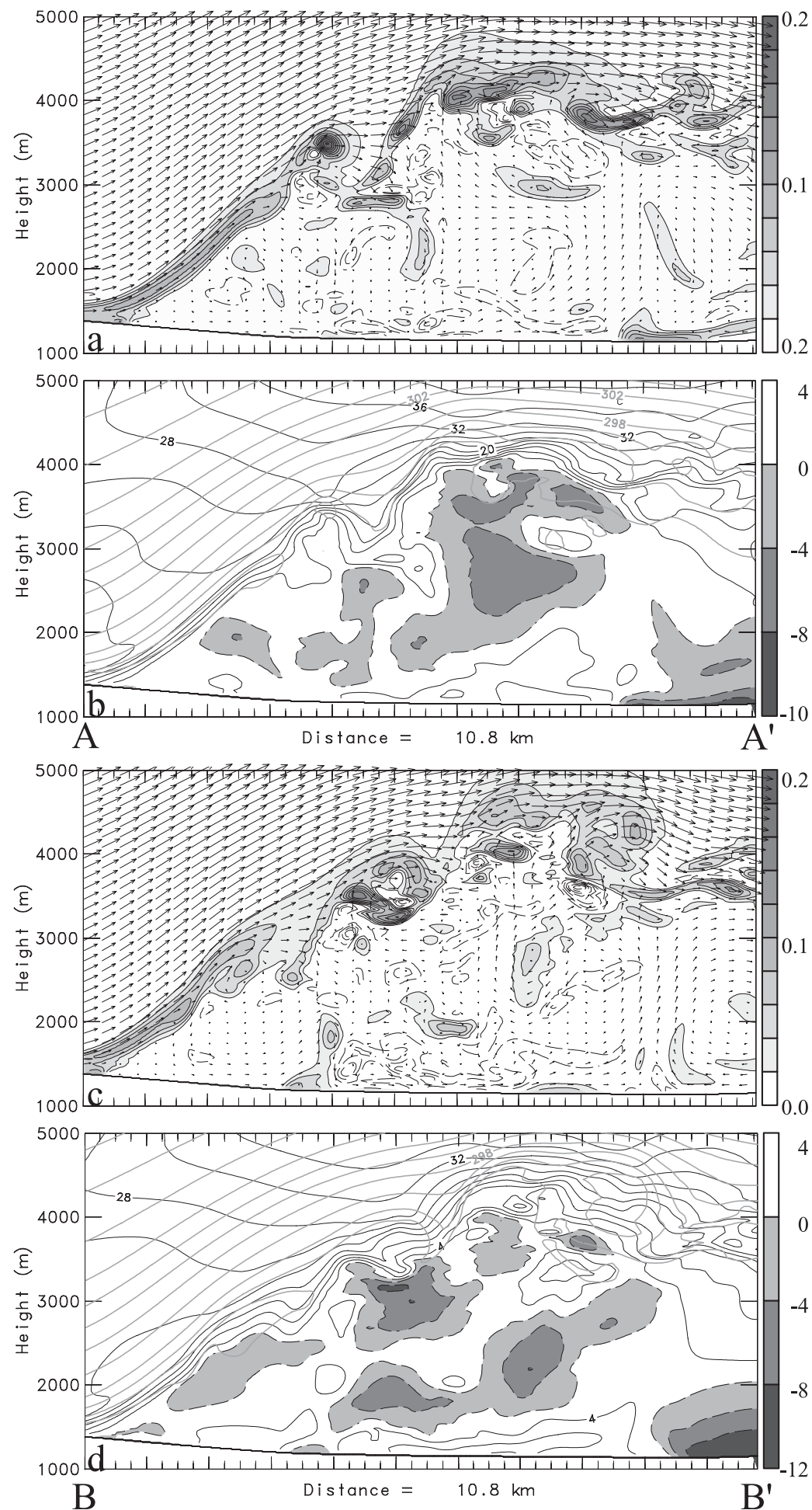

FIG. 17. (a),(b) Vertical sections of the (a) $y$-component horizontal vorticity $\left(\mathrm{s}^{-1}\right.$ ) and $(\mathrm{b})$ potential temperature $(\mathrm{K})$ and mountain-normal component wind speed $\left(\mathrm{m} \mathrm{s}^{-1}\right.$ ) for the 60-m-resolution grid mesh at $3.5 \mathrm{~h}$ along A-A' (Fig. 16). (c),(d) The (c) horizontal vorticity and (d) potential temperature and $u$-component wind speed $\left(\mathrm{m} \mathrm{s}^{-1}\right)$ for B-B' are shown at 4-h. The vorticity shading interval (positive values) and isopleths are shown every $0.02 \mathrm{~s}^{-1}, u$ wind component shading (less than zero) and isotachs are shown every $4 \mathrm{~m} \mathrm{~s}^{-1}$, and isotherms (gray contours) every $2 \mathrm{~K}$. Minor tick marks are shown every $250 \mathrm{~m}$ and major ticks every $1 \mathrm{~km}$. 
$\eta$ for the three-dimensional compressible set of equations can be expressed as

$$
\begin{aligned}
\frac{D \eta}{D t}= & -\eta\left(\frac{\partial u}{\partial x}+\frac{\partial w}{\partial z}\right)+\left[\xi \frac{\partial v}{\partial x}+(\zeta+f) \frac{\partial v}{\partial z}\right] \\
& -\left[\frac{\partial}{\partial x}(B)+C_{p}\left(\frac{\partial \theta}{\partial z} \frac{\partial \pi^{\prime}}{\partial x}-\frac{\partial \theta}{\partial x} \frac{\partial \pi^{\prime}}{\partial z}\right)\right] \\
& +\left[\frac{\partial}{\partial z}\left(D_{u}\right)-\frac{\partial}{\partial x}\left(D_{w}\right)\right],
\end{aligned}
$$

where $B$ is the buoyancy given by $g(\theta-\bar{\theta}) / \bar{\theta} ; \theta$ is the potential temperature; the $x$ - and $z$-vorticity components are $\xi$ and $\zeta$, respectively; the perturbation Exner function is $\pi^{\prime} ; c_{p}$ is the specific heat for dry air at constant pressure; and the terms $D_{u}$, and $D_{w}$ represent the subgridscale vertical mixing and horizontal smoothing terms for the $u$ and $w$ momentum equations, respectively. The first term on the rhs of the equation is the stretching term, the second represents the $y$ vorticity contribution from tilting of vorticity into the $y$ plane from other components, the third term is the baroclinic contribution, and the fourth term is the vorticity generation due to surface friction and subgrid-scale dissipation processes.

A vorticity budget shown in Fig. 20 at 3 h $35 \mathrm{~min}$ indicates that vortex stretching tends to dominate the intensification, in agreement with the simulations presented in Doyle and Durran (2007); however, contributions from tilting effects are occasionally important, particularly downstream. The $y$ component of the horizontal vorticity (Fig. 20a) indicates several strong subrotors that develop along the vortex sheet. One particularly intense subrotor, with a $0.2 \mathrm{~s}^{-1}$ vorticity maximum, is present near the center of the domain at the $3.2-\mathrm{km}$ level, approximately $3.5 \mathrm{~km}$ downstream from the left display boundary. The total Lagrangian vorticity tendency (Fig. 20b) indicates a complex pattern with a thin band of vortex intensification occurring near the subrotor of interest. The contribution from the vortex stretching (Fig. 20c) is particularly significant near the subrotor and extends downstream in a thin band with little contribution from tilting effects (Fig. 20d). Tilting contributions to the vorticity tendency are larger downstream where the turbulence becomes more three-dimensional and vorticity in other planes can be tilted into the $y$ component by the turbulent eddies. The surface friction and subgrid-scale dissipation tendency (Fig. 20e) and baroclinic terms (not shown) contribute little to the overall vorticity tendency.

Although only one example of the vorticity budget for a subrotor is shown here, examination of a number of other subrotors supports the premise that vortex stretching generally dominates the horizontal vortex in- tensification process, in agreement with the Doyle and Durran (2007) analysis. Several very strong subrotors $\left(\sim 0.35 \mathrm{~s}^{-1}\right)$ were identified in the simulation and appear to be a manifestation of a superposition of vortex stretching and tilting processes that lead to a rapid intensification of the vortex. These results differ from the Doyle and Durran study in that the vortex sheet in this case remains elevated and nearly horizontal at approximately $2.5 \mathrm{~km}$ above the valley. In contrast, the Doyle and Durran simulations indicate a tendency for the vortex sheet and vortices to descend on the downstream side of the lee wave, and in some cases the vortices reattach near the surface.

\section{c. Sensitivity to inversion strength}

An additional high-resolution 3D eddy-resolving simulation was conducted with an identical initial state, with the exception that the inversion in the $3.5-4.5-\mathrm{km}$ layer was enhanced by $2 \mathrm{~K} \mathrm{~km}^{-1}$. An east-west oriented vertical cross section of the $y$ component of horizontal vorticity, $u$ wind component, and potential temperature is shown in Fig. 21 at 3.5 h. This vertical cross section illustrates the overall sensitivity to modest changes to the upstream inversion strength. With the stronger inversion, the downslope flow strengthens to over $36 \mathrm{~m} \mathrm{~s}^{-1}$ (Fig. 21b), in contrast to $\sim 24 \mathrm{~m} \mathrm{~s}^{-1}$ in the control simulation (Figs. 17b,d), and the separation point is located farther downstream along the slopes, permitting the strong westerlies to penetrate farther into the valley. The increase in the boundary layer shear results in a vortex sheet with ambient vorticity of $0.14 \mathrm{~s}^{-1}$ (Fig. 21a), in contrast to the control simulation vortex sheet that was characterized by a $0.08 \mathrm{~s}^{-1}$ vortex sheet (Figs. 17a,c). Likewise, the shear is intensified along the vortex sheet, resulting in greater shear instability and more frequent subrotor shedding cycles. Although the subrotors generally attain more intense vorticity maxima than the control simulation, the vortex sheet exhibits less of a tendency for the vortices to remain elevated downstream, likely a consequence of changes in the amplitude and wavelength of the trapped mountain waves. The reversed flow directed back toward the Sierra beneath the lee wave crest tends to be stronger and is located primarily along the valley floor in the simulation with the enhanced inversion (Fig. 21b).

Although we have not performed a full exploration of the parameter space governing the relationship between the upstream mountain-top inversion strength and subrotors, the simulation performed with the strengthened low-level inversion does illustrate the potential sensitivity to the upstream flow conditions and the overall implications for predictability. A $2 \mathrm{~K} \mathrm{~km}^{-1}$ change in inversion strength employed in this sensitivity 

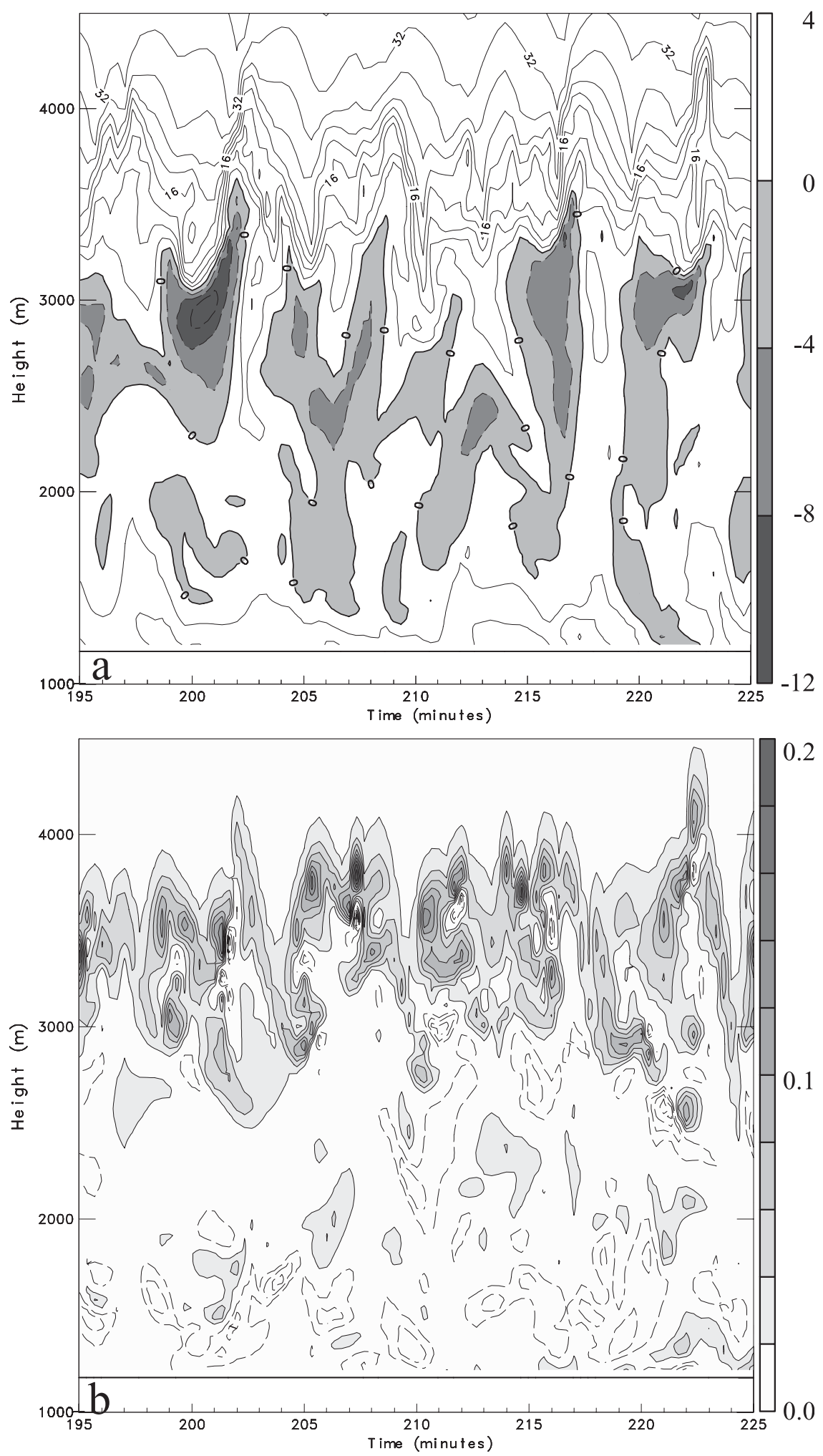

FIG. 18. Time-height section for the 3.25-3.75-h simulation times for (a) $u$ wind component $\left(\mathrm{m} \mathrm{s}^{-1}\right)$ and (b) $y$ horizontal vorticity $\left(\mathrm{s}^{-1}\right)$. The isotach interval in (a) is $4 \mathrm{~m} \mathrm{~s}^{-1}$ and the isopleth interval in (b) is $0.02 \mathrm{~s}^{-1}$. The grid point chosen displayed is $(201,101)$ on the $60-\mathrm{m}$ resolution mesh, approximately $4 \mathrm{~km}$ downstream of the separation point (for location see Fig. 16a). The $u$ wind component less than zero is shaded in (a). The vorticity greater than $0.02 \mathrm{~s}^{-1}$ is shaded in (b). 


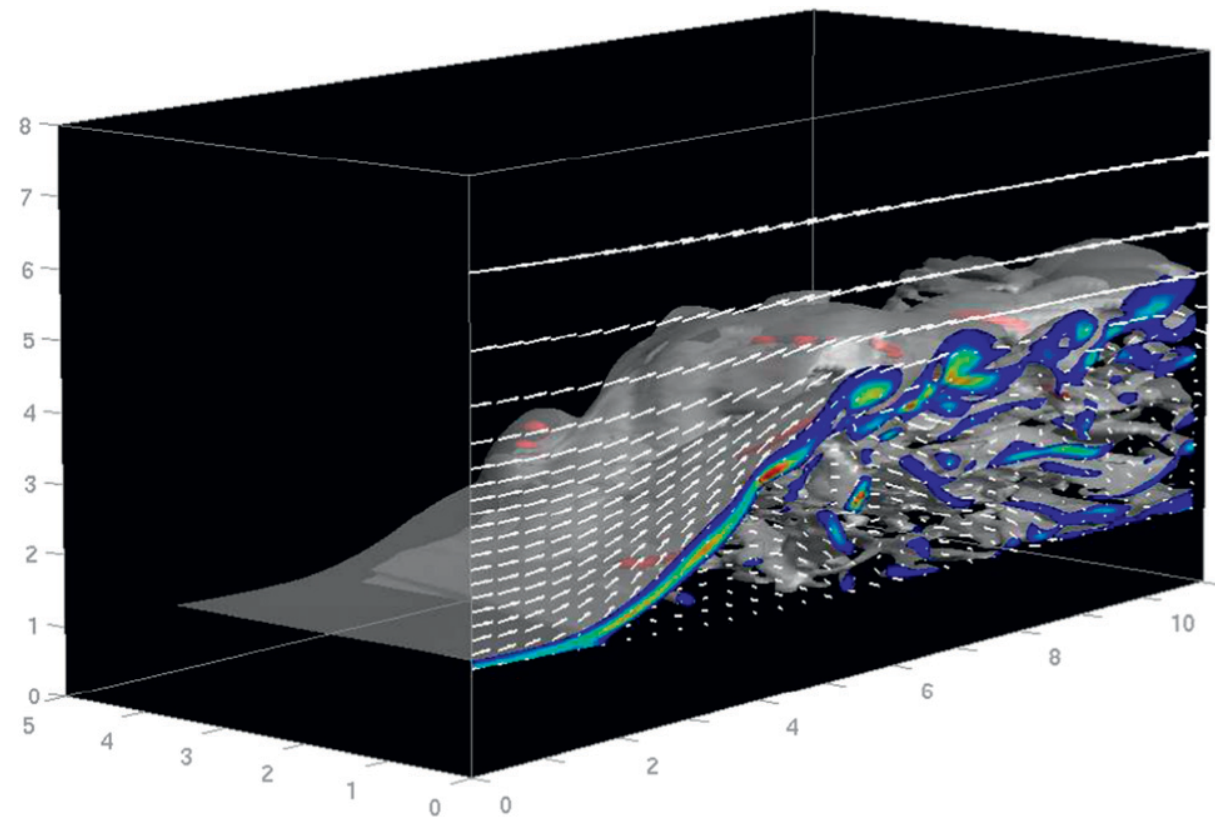

FIG. 19. Three-dimensional view of the downslope flow and subrotor vortices. The isosurface of the $0.02 \mathrm{~s}^{-1} y$ component of the horizontal vorticity is shown by the gray shading, with the red isosurface corresponding to the $0.15 \mathrm{~s}^{-1}$ vorticity. The gray isosurface that intersects the $y=0 \mathrm{~km}$ plane (at approximately $3.2 \mathrm{~km}$ or 53 grid cells from the northern edge) is shaded corresponding to positive values of the $y$ component of the horizontal vorticity ( red is $0.15 \mathrm{~s}^{-1}$ ). Wind vectors in the $y=0$ plane are shown. Distances are shown along the axes in $\mathrm{km}$.

test is likely within the bounds of numerical weather prediction errors that occur upstream of mountain barriers. The sensitivity of the turbulent subrotors to the upstream characteristics of the inversion serves to illustrate the limits to skillful prediction of mountainwave-induced rotors and associated low-level turbulence zones, particularly for high-resolution models. Similarly, it has been shown that small uncertainties in the upstream initial state within observational error limits result in limited predictability of the strength of the downslope wind speed and wave-breaking characteristics as diagnosed from idealized mesoscale ensemble forecasts (e.g., Doyle and Reynolds 2008).

\section{Conclusions}

We have explored the dynamics and internal structure of mountain-wave-induced rotors during T-REX IOP 13 through the use of a suite of ground-based and airborne observing platforms and very high-resolution simulations using a large-eddy simulation version of the nonhydrostatic COAMPS model. The observations and model results essentially confirm the existence of smallscale vortices or subrotors along a sheet of vorticity that are generated by mechanical shear in the boundary layer below strong downslope winds and lifted aloft by the lee wave circulation, in agreement with the basic characteristics of previous high-resolution threedimensional simulations (e.g., Doyle and Durran 2007). In the simulations presented here, the ascending vortex sheet becomes unstable and breaks up into in a series of subrotors along the ascending branch of the mountain lee wave.

The evolution of a very strong mountain lee wave system was documented on 16-17 April 2007 during IOP 13, which turned out to be one of the most intense IOPs during T-REX in terms of a number of metrics including turbulence strength. Ground-based instrumentation and the University of Wyoming King Air have been used to explore the turbulent characteristics of the lee wave and rotors observed during this IOP. Measurements from the King Air and satellite remote sensing imagery indicate that trapped waves were present in the lee of the Sierra on the afternoon of 16 April. The DRI surface observation network shows strong downslope winds along the western slopes, with the surface towers in the central portion of the valley indicating intermittent easterlies directed back toward the Sierra crest, which opposes the mean westerly flow. An adverse pressure gradient across the valley is also indicated by the surface observing network. The DLR Doppler and NCAR REAL aerosol lidar observations 

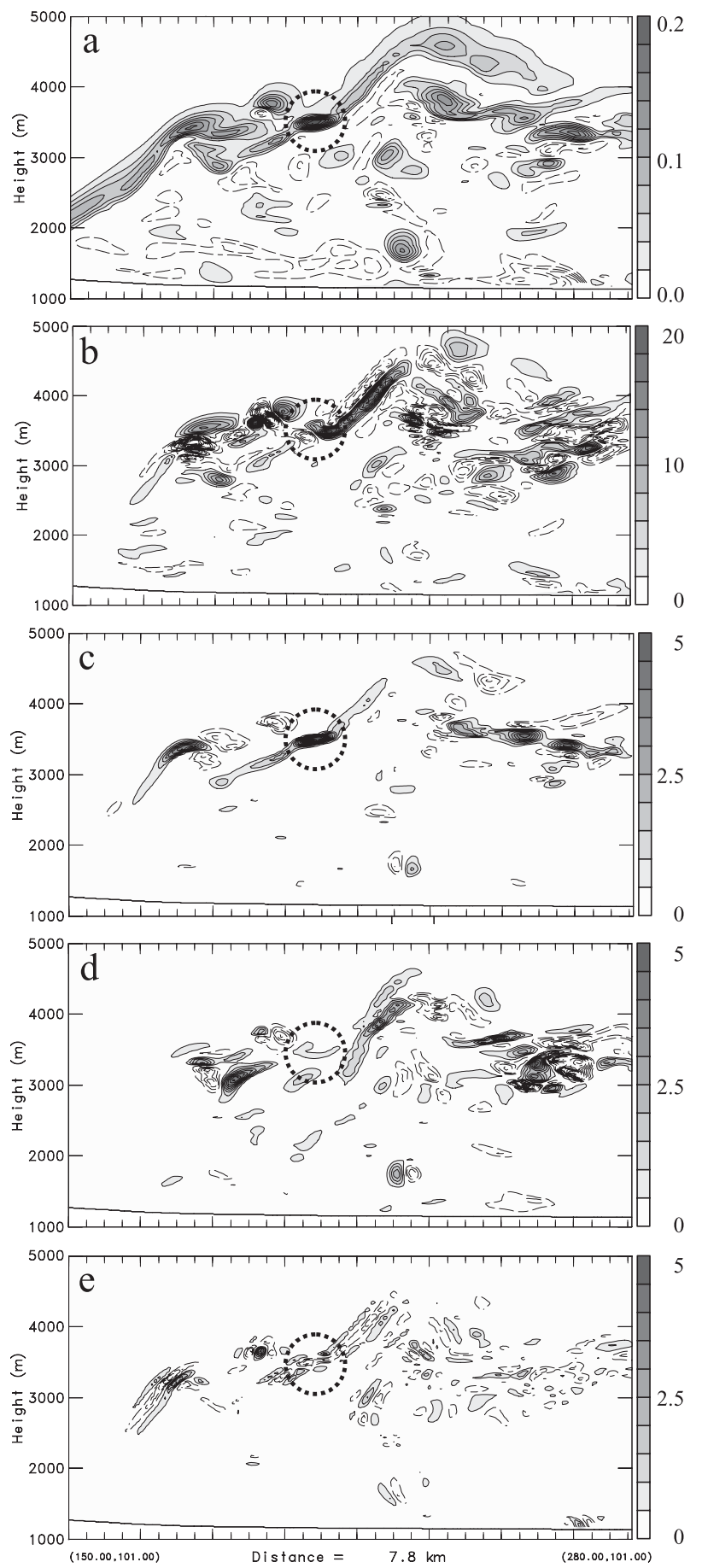

FIG. 20. Vertical section of the $y$ component horizontal vorticity budget at $3 \mathrm{~h} 35 \mathrm{~min}$. The components are (a) horizontal vorticity, (b) total vorticity tendency, (c) stretching term, (d) tilting term, and (e) surface friction and subgrid-scale dissipation terms. The shading interval shown is $0.02 \mathrm{~s}^{-1}$ in (a), $2 \times 10^{-3} \mathrm{~s}^{-2}$ in (b), and 0.5 $\times 10^{-3} \mathrm{~s}^{-2}$ in (c)-(e). Minor tick marks are shown every $250 \mathrm{~m}$ and major ticks every $1 \mathrm{~km}$. A subdomain is displayed. The dotted circles highlight the location of a subrotor of interest in (a)-(e). are used to document the life cycle of a small-scale subrotor vortex that forms over a 5-min period during 2130-2135 UTC 16 April. Downstream from the boundary layer separation region, the Doppler lidar depicts an intense shear zone associated with strong westerly flow elevated above a layer of weaker flow near the surface that is occasionally directed back toward the Sierra. Both lidars depict the evolution of an amplifying subrotor or billow that forms along the elevated shear boundary, or vortex sheet, and intensifies into a welldefined vortex, as apparent in the Doppler lidar radial velocity. The $y$ component of the horizontal vorticity derived from the lidar radial velocity exceeds $0.2 \mathrm{~s}^{-1}$ in the mature stage of the vortex, prior to being swept downstream and out of the plane of the lidar scan. The aerosol backscatter measurements are consistent with a vortex circulation embedded within a larger-scale rotor beneath the lee wave, with the upper portion of the aerosol layer overturning and folding on itself as a result of vortex amplification. Enhanced zones of aerosol backscatter occur episodically during the afternoon and evening of 16-17 April, which may be indicative of other subrotor vortex events.

The NCAR MAPR radar wind profiler also confirmed the presence of high-frequency coherent turbulent structures, consistent with the notion of subrotor vortices. The MAPR measured a series of updrafts and downdrafts with regions of enhanced reversed transverse flow during the 2115-2315 UTC time period. These turbulent vortices passed over the profiler at a frequency of $\sim 7 \mathrm{~h}^{-1}$. The high-frequency character of the flow is further illustrated by a power spectrum analysis of a surface station located near MAPR, which showed a maximum in the spectrum for frequencies in the $6-12 \mathrm{~h}^{-1}$ range. Furthermore, wavelet analysis of low-level King Air high-rate measurements exhibits a maximum at an approximate $1-\mathrm{km}$ wavelength, generally consistent with the other ground-based measurements. The cospectrum of the vertical velocity and potential temperature perturbation suggests that these large eddies were likely shear generated. Observations from ground-based observing systems and the King Air all exhibit a frequent occurrence of small-scale turbulent eddies and vortices in the 1.5-2.5-km AGL layer during this event as well as during several other mountain wave events during T-REX, suggestive that subrotors may be quite common during strongly forced mountain wave events.

High-resolution idealized simulations of IOP 13 performed in three dimensions indicate the presence of a series of subrotors that form along an elevated vortex sheet-a manifestation of a boundary layer shear zone that separates from the surface induced by the lee wave. The subrotors attain a maximum in the spanwise 


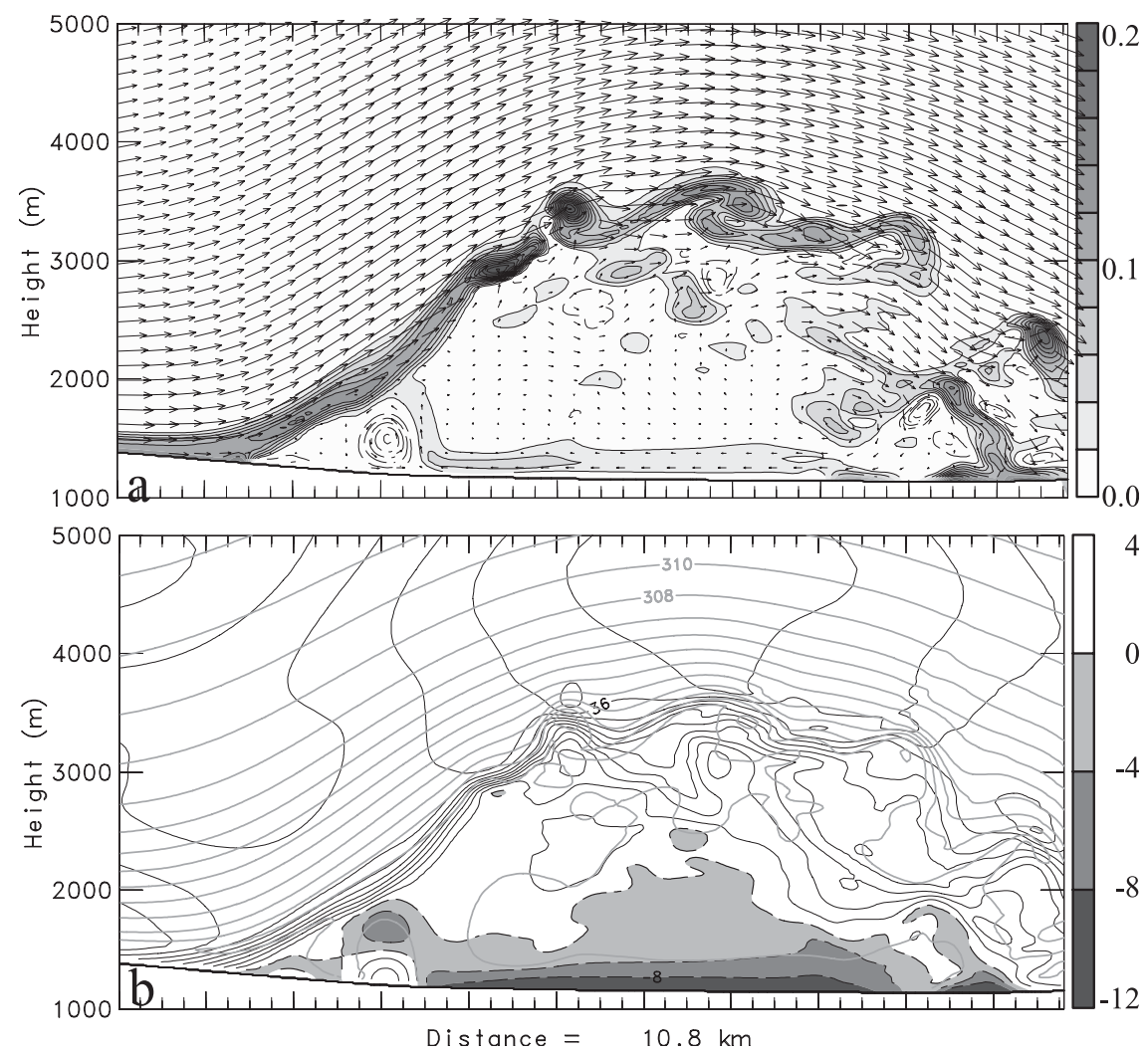

FIG. 21. Vertical sections of the (a) $y$ component horizontal vorticity $\left(\mathrm{s}^{-1}\right)$ and (b) potential temperature $(\mathrm{K})$ and mountain-normal component wind speed $\left(\mathrm{m} \mathrm{s}^{-1}\right)$ for the $60-\mathrm{m}$ resolution grid mesh at the 3.5-h time for the simulation with an enhanced temperature inversion. The vorticity shading interval (positive values) and isopleths are shown every $0.02 \mathrm{~s}^{-1}, u$ wind component shading (less than zero) and isotachs every $4 \mathrm{~m} \mathrm{~s}^{-1}$, and isotherms (gray contours) every $2 \mathrm{~K}$. Minor tick marks are shown every $250 \mathrm{~m}$ and major ticks every $1 \mathrm{~km}$.

vorticity of $\sim 0.2 \mathrm{~s}^{-1}$, similar to the horizontal vorticity derived from the DLR lidar. The subrotors detach from the vortex sheet and are swept downstream, creating an elevated zone of turbulence and vortices approximately $1.5-2.5 \mathrm{~km}$ above the valley. Intensification of the subrotor vortices occurs as they break away from the vortex sheet through vortex stretching and in some cases tilting processes related to three-dimensional turbulent mixing. The ambient vortex sheet and subrotors intensify in simulations that contain a modest increase in the upstream mountain-top inversion strength. However, in the simulation with increased inversion strength, vortices exhibit less of a tendency to remain elevated downstream, which illustrates the predictability challenges for the turbulence characterization of rotors.

The observations and simulations demonstrate that subrotor vortices likely pose a substantial hazard to aviation because of the associated strong wind shear and transient nature of the circulations. On 3 March 1991, a B737 crashed at the Colorado Springs, Colorado, air- port and all 25 passengers and crew onboard perished. As part of the National Transportation Safety Board (NTSB) investigation, engineers and scientists considered the possibility of the crash resulting from strong wind shear and rotors, which were observed to be present that day (National Transportation Safety Board 1992). Engineers found that translating rotors with horizontal vorticity of $0.8 \mathrm{~s}^{-1}$ or greater resulted in crash scenarios using B737 flight simulators. One of the fundamental questions arising from the NTSB report is related to the potential strength of rotor circulations. The T-REX observations and related numerical simulations suggest that subrotors can attain maximum vorticity of $0.2 \mathrm{~s}^{-1}$, or even larger under some conditions $\left(\sim 0.4 \mathrm{~s}^{-1}\right)$, and indeed may pose a serious threat to low-level flying aircraft.

Acknowledgments. Dale Durran and Joachim Kuettner are gratefully acknowledged for helpful discussions. The efforts of the National Center for Atmospheric 
Research (NCAR) Field Project Support (FPS) and the T-REX staff are greatly appreciated. The primary sponsor of T-REX is the U.S. National Science Foundation (NSF). We acknowledge also other funding agencies and organizations that have provided funding and inkind support for various T-REX components including the UK Natural Environmental Research Council (NERC), Met Office UK, U.S. Naval Research Laboratory (NRL), National Oceanic and Atmospheric Administration (NOAA), Deutsches Zentrum für Luft- und Raumfahrt (DLR), Swiss National Science Foundation (SNSF), and the Austrian Science Foundation (FWF). The first and second author, respectively, acknowledge support through ONR PE 0601153N and NSF ATM0524891. COAMPS is a registered trademark of NRL. The support of Andreas Wieser for DLR lidar observations and lidar analysis software from Ron Calhoun and Christian Kühnlein is acknowledged. The Doppler lidar operated by DLR was leased from Lockheed Martin Coherent Technologies and their technical support is acknowledged. We thank Saša Gaberšek for helpful discussions and graphical contributions.

\section{REFERENCES}

Banta, R. M., L. D. Olivier, and J. M. Intrieri, 1990: Doppler lidar observations of the 9 January 1989 severe downslope windstorm in Boulder, Colorado. Preprints, Fifth Conf. on Mountain Meteorology, Boulder, CO, Amer. Meteor. Soc., 68-69.

Bond, N. A., C. F. Dierking, and J. D. Doyle, 2006: Research aircraft and wind profiler observations in Gastineau Channel during a Taku wind event. Wea. Forecasting, 21, 489-501.

Bougeault, P., 1983: A non-reflective upper boundary condition for limited-height hydrostatic models. Mon. Wea. Rev., 111, 420-429.

Carney, T. Q., A. J. Bedard Jr., J. M. Brown, J. McGinley, T. Lindholm, and M. J. Kraus, 1996: Hazardous mountain winds and their visual indicators. Federal Aviation Administration Advisory Circular AC00-57, $80 \mathrm{pp}$.

Cohn, S. A., W. O. J. Brown, C. L. Martin, M. E. Susedik, G. Maclean, and D. B. Parsons, 2001: Clear air boundary layer spaced antenna wind measurement with the Multiple Antenna Profiler (MAPR). Ann. Geophys., 19, 845-854.

Colson, D., 1954: Meteorological problems in forecasting mountain waves. Bull. Amer. Meteor. Soc., 35, 363-371.

Cuxart, J., G. Morales, E. Terradellas, and C. Yagüe, 2002: Study of coherent structures and estimation of the pressure transport terms for the nocturnal stable boundary layer. Bound.Layer Meteor., 105, 305-328.

Darby, L. S., and G. S. Poulos, 2006: The evolution of lee-waverotor activity in the lee of Pike's Peak under the influence of a cold frontal passage: Implications for aircraft safety. Mon. Wea. Rev., 134, 2857-2876.

De Wekker, S. F. J., and S. D. Mayor, 2009: Observations of atmospheric structure and dynamics in the Owens Valley of California with a ground-based, eye-safe, scanning aerosol lidar. J. Appl. Meteor. Climatol., in press.
Doyle, J. D., and D. R. Durran, 2002: The dynamics of mountainwave-induced rotors. J. Atmos. Sci., 59, 186-201.

$\longrightarrow$, and — 2007: Rotor and subrotor dynamics in the lee of three-dimensional terrain. J. Atmos. Sci., 64, 4202-4221.

—_, and C. A. Reynolds, 2008: Implications of regime transitions for mountain-wave-breaking predictability. Mon. Wea. Rev., 136, 5211-5223.

Durran, D. R., 1990: Mountain waves and downslope winds. Atmospheric Processes over Complex Terrain, B. Blumen Ed., Amer. Meteor. Soc., 59-81.

_, 1999: Numerical Methods for Wave Equations in Geophysical Fluid Dynamics. Springer-Verlag, 465 pp.

—, M.-J. Yang, D. N. Slinn, and R. G. Brown, 1993: Toward more accurate wave-permeable boundary conditions. Mon. Wea. Rev., 121, 604-620.

Fritts, D. C., T. L. Palmer, Ø. Andreassen, and I. Lie, 1996: Evolution and breakdown of Kelvin-Helmholtz billows in stratified compressible flows. Part I: Comparison of two- and threedimensional flows. J. Atmos. Sci., 53, 3173-3191.

Golaz, J.-C., S. Wang, J. D. Doyle, and J. M. Schmidt, 2005: COAMPS-LES: Model evaluation and analysis of second-and third-moment vertical velocity budgets. Bound.-Layer Meteor., 116, $487-517$

Grubišić, V., and J. M. Lewis, 2004: Sierra Wave Project revisited: 50 years later. Bull. Amer. Meteor. Soc., 85, 1127-1142.

- , and B. J. Billings, 2007: The intense lee-wave rotor event of Sierra Rotors IOP 8. J. Atmos. Sci., 64, 4178-4201.

- , and I. Stiperski, 2009: Lee-wave resonances over double bellshaped obstacles. J. Atmos. Sci., 66, 1205-1228.

— ment: A field campaign overview including observational highlights. Bull. Amer. Meteor. Soc., 89, 1513-1533.

Hertenstein, R. F., and J. P. Kuettner, 2005: Rotor types associated with steep lee topography: Influence of the wind profile. Tellus, 57A, 117-135.

Hodur, R. M., 1997: The Naval Research Laboratory's Coupled Ocean/Atmosphere Mesoscale Prediction System (COAMPS). Mon. Wea. Rev., 125, 1414-1430.

Holmboe, J., and H. Klieforth, 1957: Investigations of mountain lee waves and airflow over the Sierra Nevada. Final Rep., Contract AF19(604)-728, Dept. of Meteorology, University of California, Los Angeles, 290 pp.

Jiang, Q., and J. D. Doyle, 2008: Diurnal variation of downslope winds in Owens Valley during the Sierra Rotor Experiment. Mon. Wea. Rev., 136, 3760-3780.

Kahn, B. H., W. Chan, and P. F. Lester, 1997: An investigation of rotor flow using DFDR data. Proc. Seventh Conf. on Aviation, Range, and Aerospace Meteorology, Long Beach, CA, Amer. Meteor. Soc., 206-210.

Klaassen, G. P., and W. R. Peltier, 1985: Evolution of finite amplitude Kelvin-Helmholtz billows in two spatial dimensions. J. Atmos. Sci., 42, 1321-1339.

Klemp, J., and R. Wilhelmson, 1978: The simulation of three-dimensional convective storm dynamics. J. Atmos. Sci., 35, 1070-1096. , and D. R. Durran, 1983: An upper boundary condition permitting internal gravity wave radiation in numerical mesoscale models. Mon. Wea. Rev., 111, 430-444.

Kuettner, J., 1959: The rotor flow in the lee of mountains. GRD Research Note 6, Air Force Cambridge Research Center, 20 pp.

Lester, P. F., and W. A. Fingerhut, 1974: Lower turbulent zones associated with mountain lee waves. J. Appl. Meteor., 13, 54-61.

Lilly, D. K., 1962: On the numerical simulation of buoyant convection. Tellus, 14, 148-172. 
Louis, J.-F., 1979: A parametric model of vertical eddy fluxes in the atmosphere. Bound.-Layer Meteor., 17, 187-202.

— M. Tiedtke, and J. F. Geleyn, 1982: A short history of the operational PBL parameterization at ECMWF. Proc. Workshop on Planetary Boundary Layer Parameterization, Reading, United Kingdom, ECMWF, 59-79.

Mayor, S. D., S. M. Spuler, B. M. Morley, and E. Loew, 2007: Polarization lidar at $1.54 \mu \mathrm{m}$ and observations of plumes from aerosol generators. Opt. Eng., 46, 096201, doi:10.1117/1.2786406.

Mobbs, S. D., and Coauthors, 2005: Observations of downslope winds and rotors in the Falkland Islands. Quart. J. Roy. Meteor. Soc., 131, 329-351, doi:10.1256/qj.04.51.

National Transportation Safety Board, 1992: Aircraft accident report: Colorado Springs, CO, 3 March 1991. AAR-92-06, PB92-910407, 160 pp.
Orlanski, I., 1976: A simple boundary condition for unbounded hyperbolic flows. J. Comput. Phys., 21, 251-269.

Pearson, R. A., 1974: Consistent boundary conditions for numerical models of systems that admit dispersive waves. J. Atmos. Sci., 31, 1481-1489.

Vosper, S. B., 2004: Inversion effects on mountain lee waves. Quart. J. Roy. Meteor. Soc., 130, 1723-1748.

Weissmann, M. D., G. J. Mayr, R. M. Banta, and A. Gohm, 2004: Observations of the temporal evolution and spatial structure of the gap flow in the Wipp Valley on 2 and 3 October 1999. Mon. Wea. Rev., 132, 2684-2697.

— A. Aörnbrack, and J. D. Doyle, 2009: Vorticity from line-ofsight lidar velocity scans. J. Atmos. Oceanic Technol., submitted.

Whelan, R. F., 2000: Exploring the Monster: Mountain Lee Waves: The Aerial Elevator. Wind Canyon Books, 169 pp. 